\title{
Preliminary Comparison of Reaction Rate Theory and Object Kinetic Monte Carlo Simulations of Defect Cluster Dynamics under Irradiation
}

S. I. Golubov, R. E. Stoller, C. S. Becquart, and C. Domain

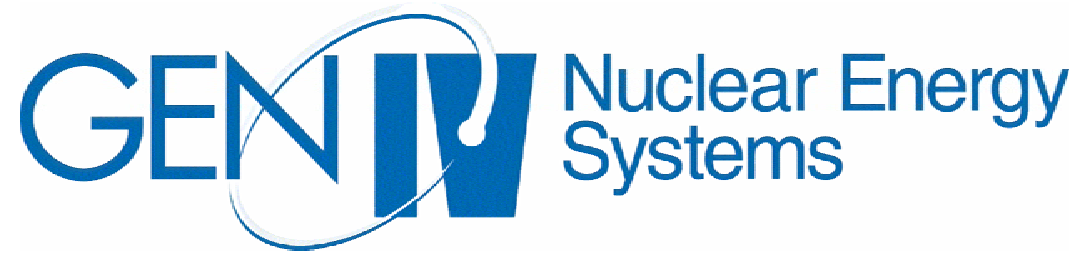

September 22, 2006

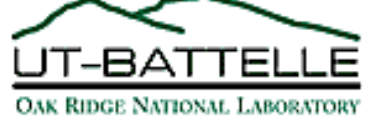


This report was prepared as an account of work sponsored by an agency of the United States Government. Neither the United States government nor any agency thereof, nor any of their employees, makes any warranty, express or implied, or assumes any legal liability or responsibility for the accuracy, completeness, or usefulness of any information, apparatus, product, or process disclosed, or represents that its use would not infringe privately owned rights. Reference herein to any specific commercial product, process, or service by trade name, trademark, manufacturer, or otherwise, does not necessarily constitute or imply its endorsement, recommendation, or favoring by the United States Government or any agency thereof. The views and opinions of authors expressed herein do not necessarily state or reflect those of the United States Government or any agency thereof. 


\title{
Preliminary Comparison of Reaction Rate Theory and Object Kinetic Monte Carlo Simulations of Defect Cluster Dynamics under Irradiation
}

\author{
S. I. Golubov, R. E. Stoller \\ Materials Science and Technology Division \\ Oak Ridge National Laboratory \\ C. S. Becquart \\ Université de Lille I, 59655 Villeneuve D'Ascq, France \\ C. Domain \\ EDF-R\&D, Dept. MMC, Les Renardières, Moret-sur-Loing, France \\ September 22, 2006 \\ Prepared for \\ Office of Nuclear Energy Science and Technology \\ Prepared by \\ OAK RIDGE NATIONAL LABORATORY \\ Oak Ridge, Tennessee 37831 \\ managed by \\ UT-BATTELLE, LLC \\ for the \\ U.S. DEPARTMENT OF ENERGY \\ Under DOE Contract No. DE-AC05-00OR22725
}




\begin{abstract}
ABTRACT
The multiscale modeling scheme encompasses models from the atomistic to the continuum scale. Phenomena at the mesoscale are typically simulated using reaction rate theory (RT), Monte Carlo (MC), or phase field models. These mesoscale models are appropriate for application to problems that involve intermediate length scales ( $\mu \mathrm{m}$ to $>\mathrm{mm})$, and timescales from diffusion $(\sim \mu \mathrm{s})$ to long-term microstructural evolution ( $\sim$ years). Phenomena at this scale have the most direct impact on mechanical properties in structural materials of interest to nuclear energy systems, and are also the most accessible to direct comparison between the results of simulations and experiments. Recent advances in computational power have substantially expanded the range of application for MC models. Although the RT and MC models can be used simulate the same phenomena, many of the details are handled quite differently in the two approaches. A direct comparison of the RT and MC descriptions has been made in the domain of point defect cluster dynamics modeling, which is relevant to both the nucleation and evolution of radiation-induced defect structures. The relative merits and limitations of the two approaches are discussed, and the predictions of the two approaches are compared for specific irradiation conditions.
\end{abstract}




\section{CONTENTS}

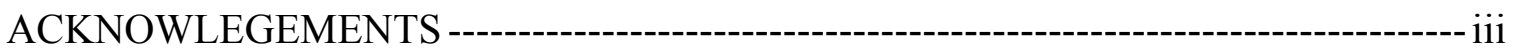

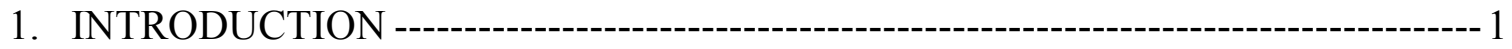

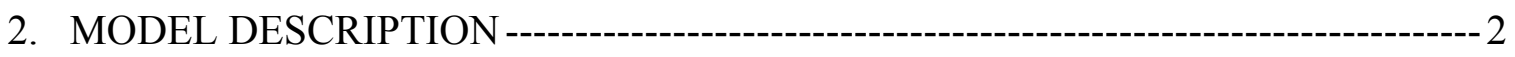

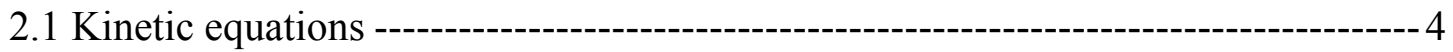

2.2 Initial and boundary conditions --------------------------------------------------------------- 8

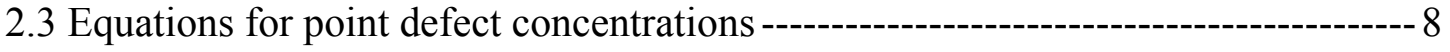

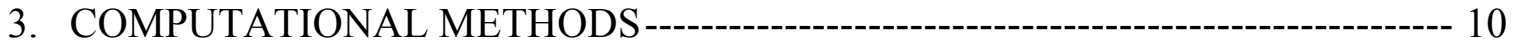

3.1 The grouping method for approximate numerical solution of ME ------------------ 10

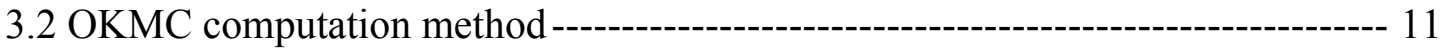

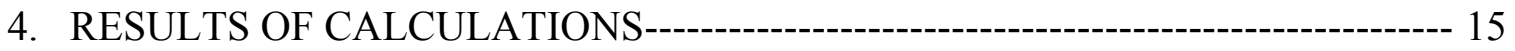

4.1 Cluster evolution at Frenkel pair production at 373K ----------------------------- 17

4.2 Comparison of results obtained by use of RT and OKMC methods --------------- 24

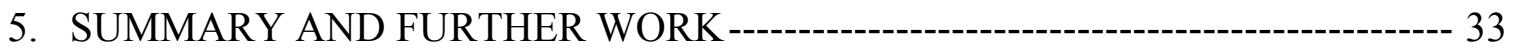

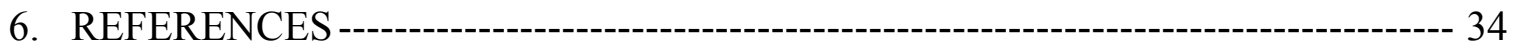




\section{ACKNOWLEDEMENTS}

The ORNL authors are grateful to their French colleagues who provided the OKMC expertise to make this comparison possible, and to William Corwin for programmatic direction and constructive comments.

This work is sponsored by the U.S. Department of Energy, Office of Nuclear Energy Science and Technology under contract DE-AC05-00OR22725 with Oak Ridge National Laboratory, managed by UT-Battelle, LLC. 


\section{INTRODUCTION}

In many important cases, changes in the physical and mechanical properties of materials during thermal aging or under irradiation occur because of the formation and growth of point defect clusters (e.g. voids and dislocation loops) and second-phase precipitates. In order to understand these changes quantitatively, it is important to have a method for describing the evolution of these clusters covering all stages from their nucleation to growth and coarsening. If a random spatial distribution of the defect clusters is assumed, the microstructural evolution can be described in terms of a defect size distribution function (SDF) in the framework of the socalled mean field approximation. Evolution of this SDF can be accounted for though the use of a Master Equation (ME) that describes both growth and dissolution of the clusters due to reactions with mobile defects (or solutes), thermal emission of these same species, and cluster coalescence if the clusters are mobile. The relevant physical processes require accounting for clusters containing a very large number of point defects or atoms $\left(>10^{6}\right)$, leading to a system with the same number of differential equations in which time is an explicit variable. Such calculations are time consuming and, especially for high irradiation doses or long ageing times and can be performed only if the number of equations is reduced significantly. Practical solutions to the ME by numerical methods employ some grouping procedure to significantly reduce the number of equations [1-4].

The object kinetic Monte Carlo technique (OKMC) is another way to calculate cluster evolution. This method has become practicable during last decade because of progress in computer technology. In the framework of OKMC, each point defect or defect cluster is treated as an object located in a specific position in the simulation box. Irradiation is simulated by introducing new point defects or defect clusters at discrete times and in specific locations in the box, and evolution occurs as the various objects migrate and participate in a series of predefined reactions with other objects. Each object has an associated reaction volume. The reaction probabilities for physical transition mechanisms, which are basically migration jump rates and defect emission rates from larger defects or from traps, are calculated based on Arrhenius frequencies for thermally activated events. The Monte Carlo algorithm [5] is used at each step to select the event that is going to take place based on the corresponding probabilities. After a given 
event is chosen, the time is increased according to a algorithm that depends on the jump frequencies for all the possible events in the system.

An advantage of the ME approach is there are essentially no limits to the density or size of the clusters when calculating their evolution, which provides the opportunity to compare with a broad range of experimental observations. However, the spatial and time correlations in defect production are not accounted for, which in some cases lead to a loss of specific information. OKMC on the other hand is capable of accounting for these spatial and time correlations in defect production, although limitations can arise related to the available computer power. The main limitation is on the size of the simulation cell in order to maintain a reasonable simulation time. The practicable box size, which normally is taken to be of order of $100 \mathrm{~nm}$, limits the total cluster number density which may be calculated by this method. For example, if there is only one cluster in a cubic box with a $100 \mathrm{~nm}$ edge length, the corresponding to cluster number density is $10^{21} \mathrm{~m}^{-3}$. This is a typical defect density for many relevant irradiated materials, and no lower cluster density can be treated by OKMC in a box of this size. Even for the relatively high defect density of $10^{22} \mathrm{~m}^{-3}$, the number of clusters in the simulation box would only be 10 , which may not be enough to provide a good statistical simulation of defect cluster evolution. Another problem with statistics may arise when simulating clusters which are not stable in a certain size range, i.e. when cluster nucleation requires reaching a certain critical size at which they become stable enough to be able grow continuously. Such nucleation is possible due to fluctuations in the cluster growth and resolution processes and will be properly calculated by OKMC only if the density is sufficiently high to maintain such fluctuations.

Thus, both the RT and OKMC have advantages and limitations based on the underlying nature of the technique; the major features of these models are briefly summarized in Table 1. Perhaps the best way to assess these methods is to apply both of them to a well-defined problem and determine if the results are consistent between the two, or if the results indicate that one of the other is preferable. In order to reach this point, the limitations have to be systematically studied and this is the main objective of the present study. Since the two techniques provide two different mathematical ways of solving the same problem, they should provide similar results for well designed sample problems. Since the RT approach has been very well established by broad use during last few decades, the approach taken in this present work was to use RT calculations 
as a reference point to evaluate the predictions of the OKMC method for several different types of simulations. The RT model used for this comparison is presented in the next section, and a more detailed description of both techniques is presented in Section 3. The results of the calculations and a short summary of the conclusions are given in Sections 4 and 5, respectively.

Table 1: Comparison of reaction rate theory and object kinetic Monte Carlo methods

\begin{tabular}{|c|c|c|}
\hline $\begin{array}{c}\text { Feature or Treatment of } \\
\text { Variable }\end{array}$ & Rate Theory & Object Kinetic Monte Carlo \\
\hline solution method & deterministic & stochastic \\
\hline time & explicit variable & $\begin{array}{l}\text { inferred from possible } \\
\text { processes and reaction rates }\end{array}$ \\
\hline space & $\begin{array}{l}\text { homogeneous effective medium, } \\
\text { possible multi-region RT }\end{array}$ & full spatial dependence \\
\hline defect production & $\begin{array}{l}\text { time and space-averaged, but } \\
\text { c.f. cascade diffusion model [6] }\end{array}$ & discrete in time and space \\
\hline sink strength & $\begin{array}{l}\text { explicit input parameter or } \\
\text { analytical expression }\end{array}$ & $\begin{array}{l}\text { inferred from fate of point } \\
\text { defects }\end{array}$ \\
\hline defect or sink density & essentially unlimited & $\begin{array}{l}\text { limited (computationally) by } \\
\text { simulation cell size, i.e.: } \\
\mathrm{N}_{\min } \geq 1 / \text { (box volume) }\end{array}$ \\
\hline
\end{tabular}

\section{MODEL DESCRIPTION}

The comparison of the two methods is more straight forward if we consider a particular case. Here we will investigate the evolution of the size distribution function of vacancy and selfinterstitial atoms (SIAs) clusters in a pure metal under irradiation with network dislocations as a fixed sink. in the following discussion, the term void is used in a generic way to refer to a vacancy cluster of any size. The formulated model makes use of the following assumptions:

1. The primary damage is produced either: (a) in the form of Frenkel pair, i.e. only single vacancies and SIAs; or (b) Frenkel pair plus small clusters of either vacancies or SIAs generated by cascades

2. The point defects execute a three-dimensional random walk 
3. The nucleation of vacancy and SIA clusters proceeds via a homogeneous mechanism, that is due to monodefect + monodefect $=$ di-defect in the case of $1(\mathrm{a})$, or by both homogeneous and in-cascade clustering mechanisms at the same time for 1(b).

4. Vacancy clusters are treated as spherical voids that are neutral sinks for point defects, and with a size-dependent stability against thermal vacancy emission

5. SIA clusters are treated as either planar loops or three dimensional spherical clusters, with a preference (bias) for absorbing SIAs relative to vacancies, and are stable against thermal SIA emission

6. Edge dislocations are a fixed matrix sink with a preference (bias) for SIAs that is the same as the SIA clusters.

7. The vacancy and SIA clusters are immobile.

These assumptions are only used for simplification and do not lead to any restrictions in comparing the two methods.

\subsection{Kinetic equations}

Essentially, in the case when the both techniques may be used for the calculations a set of equations describing cluster evolution is the same. Having this in minds, the set of equations for the case under consideration is formulated within the framework of RT.

For the model described above, one can write the following Master Equations for the SDF of voids, $f_{v}(x, t)$, and SIA clusters, $f_{i}(x, t)$, where $x$ is the number of point defects in the cluster $[7$, 8].

$$
\begin{gathered}
\frac{\partial f_{v}(x, t)}{\partial t}=K_{v}(x)+J_{v}(x-1, t)-J_{v}(x, t), \\
\frac{\partial f_{i}(x, t)}{\partial t}=K_{i}(x)+J_{i}(x-1, t)-J_{i}(x, t), \\
\sum_{x=2}^{\infty} x K_{v}(x)=\varepsilon_{v} G_{N R T}\left(1-\varepsilon_{r}\right), \sum_{x=2}^{\infty} x K_{i}(x)=\varepsilon_{i} G_{N R T}\left(1-\varepsilon_{r}\right),
\end{gathered}
$$

where $K_{v}(x), K_{i}(x)$ are the rates of in-cascade generation of voids and SIA loops, respectively, $\varepsilon_{r}$ is the fraction of Frenkel pair which recombine during the cascade cooling, $\varepsilon_{v}, \varepsilon_{i}$ are the in- 
cascade clustering fractions for vacancies and SIAs, respectively, $G_{N R T}$ is the NRT Frenkel pair generation rate, and $J_{v}(x, t), J_{i}(x, t)$ are the void and SIA loop fluxes in cluster size space, respectively.

$$
\begin{gathered}
J_{v}(x, t)=P_{v}(x, t) f(x, t)-Q_{v}(x+1, t) f(x+1, t), \\
J_{i}(x, t)=P_{i}(x, t) f(x, t)-Q_{i}(x+1, t) f(x+1, t) .
\end{gathered}
$$

In Eqs. (2), $P_{v}(x, t)$ and $P_{i}(x, t)$ are the rates of vacancy absorption by a void and SIA absorption by a SIA cluster, respectively; $Q_{v}(x, t)$ is the sum of the rates of SIA absorption and vacancy emission from a void, and $Q_{i}(x, t)$ the rate of vacancy absorption by a SIA cluster. These rates depend on the cluster concentration and the diffusion properties of the mobile defects, i.e. vacancies and SIAs. In the case of 3-D diffusion of point defects to voids and SIA loops the rates take the following form $[7,8]$ :

$$
\begin{gathered}
P_{v}(x)=w_{v} x^{1 / 3} D_{v} C_{v}(t), \\
Q_{v}(x)=w_{v} x^{1 / 3}\left[D_{i} C_{i}+D_{v} \exp \left(-E_{v}^{b}(x) / k T\right)\right]=Q_{v}^{i}(x)+Q_{v}^{v}(x), \\
P_{i}(x)=Z_{i}^{l} w_{i} x^{1 / 2} D_{i} C_{i}(t), \\
Q_{i}(x)=Z_{v}^{l} w_{i} X^{1 / 2} D_{v} C_{v}(t), \\
w_{v}=\left(\frac{48 \pi^{2}}{\Omega^{2}}\right)^{1 / 3}, w_{i}=\left(\frac{4 \pi}{\Omega b}\right)^{1 / 2} .
\end{gathered}
$$

$C_{i, v}(t)$ and $D_{i, v}$ are the concentrations and diffusion coefficients, respectively, of vacancies (subscript v) and SIAs (subscript i), $Z_{v}^{l}, Z_{i}^{l}$ are the dislocation loop capture efficiencies for vacancies and SIAs, $E_{v}^{b}(x)$ is the binding energy of a vacancy to a vacancy cluster of size $\mathrm{x}, k_{B}$ is Boltzmann's constant, $\mathrm{T}$ is the absolute temperature, $\Omega$ is the atomic volume, and $\mathrm{b}$ is the magnitude of the SIA loop Burgers vector. In the case when the dislocation loops are treated as $3-\mathrm{D}$ clusters, which is used in the OKMC calculations, the parameter $w_{i}$ has to be replaced by $w_{v}$ and $x^{1 / 2}$ replaced by of $x^{1 / 3}$ in Eqs. (4a) and (4b).

Note that if the SDFs described by Eqs. (1a) and (1b) are written in x-space, the cluster densities are dimensionless. That is, the total density of clusters, $N_{v, i}=\sum_{x=2}^{\infty} f_{v, i}(x)$, has the units 
[1/atom]. The capture efficiencies given by Eqs. (3) and (4) have dimensions of $\left[\mathrm{m}^{-2}\right]$, and the cluster sink strengths, which are given by

$$
\begin{aligned}
k_{v}^{2} & =\sum_{x=2}^{\infty} P_{v}(x) f_{v}(x) \equiv w_{v} \sum_{x=2}^{\infty} x^{1 / 3} f_{v}(x), \\
k_{i v, i}^{2} & =\sum_{x=2}^{\infty} P_{i}(x) f_{i}(x) \equiv Z_{v, i} w_{i} \sum_{x=2}^{\infty} x^{1 / 2} f_{i}(x),
\end{aligned}
$$

have the same dimensionality of $\left[\mathrm{m}^{-2}\right]$. For the purpose of comparing the results obtained by the two different techniques, it will be useful to show that the sink strengths given by Eqs. (6) are fully equivalent to those that are normally used in the mean size approximation:

$$
k_{v, v, i}^{2}=4 \pi<R_{v}>N_{v}, k_{i, v, i}^{2}=2 \pi Z_{v, i}^{l}<R_{i}>N_{i},
$$

which describe damage accumulation in the form of voids and loops by using the mean cluster radii $\left\langle\mathrm{r}_{v}\right\rangle,\left\langle r_{i}\right\rangle$ and the corresponding total number densities $N_{v}, N_{i}$ in the units of $m$ and $m^{-3}$, respectively. In order to show this, the SDF can be calculated in the domain where the size of a cluster is defined by its radius, $r$, instead of $\mathrm{x}$. The SDFs can be calculated by taking into account that a sum of the SDF over all sizes in any phase space used for the ME has to be equal to the total number of clusters, $N_{\text {tot }}$. Replacing the sums with integrals, the total number density of the clusters in the case under consideration may be written as

$$
N_{\text {tot }}=\int_{x=2}^{\infty} f(x) d x \equiv \int_{R=R_{\min }}^{\infty} f(R) d R .
$$

The two integral can be equal to each other if

$$
f(x) d x=f(R) d R .
$$

Taking into account that the radii of voids, $r_{v}$, and loops, $r_{i}$, and the total number of defects in the clusters, $x_{v}, x_{i}$, related to each other as follows

$$
\frac{4 \pi}{3} R_{v}^{3}=x_{v} \Omega, \pi R_{i}^{2} b=x_{i} \Omega,
$$

and using Eq. (9) the functions $f_{v}\left(r_{v}\right)$ and $f_{i}\left(r_{i}\right)$ can be easily calculated

$$
f_{v}\left(R_{v}\right)=f_{v}(x)\left(\frac{36 \pi}{\Omega}\right)^{1 / 3} x^{2 / 3}, \quad f_{i}\left(R_{i}\right)=f_{i}(x)\left(\frac{4 \pi b}{\Omega}\right)^{1 / 2} x^{1 / 2}
$$


Note that dimensionality of the functions $f_{v}\left(R_{v}\right), f_{i}\left(R_{i}\right)$ is $\left[\frac{1}{m} \frac{1}{a t o m}\right]$. On the other hand the SDFs in r-space are normally presented as a number of clusters per unit volume. This can be obtained by dividing the right hand sides of Eqs. (11) by the atomic volume, $\Omega$, i.e. $F_{v, i}\left(R_{v, i}\right)=\frac{1}{\Omega} f_{v, i}\left(R_{v, i}\right)$. Here the capital $\mathrm{F}$ is used to distinguish between the dimensional SDF

$\left(1 / \mathrm{m}^{4}\right)$ and SDFs $\left.f_{v}\left(R_{v}\right), f_{i}\left(R_{i}\right)\right)$. Using Eqs. (10), (11) and replacing the sums in Eq. (6) by the corresponding integrals one can easily find that:

$$
\begin{gathered}
k_{v}^{2}=\left(\frac{48 \pi^{2}}{\Omega^{2}}\right)^{1 / 3} \int_{x=2}^{\infty} x^{1 / 3} f_{v}(x) d x=4 \pi \int_{R_{v, \text { min }}}^{\infty} R_{v} F_{v}\left(R_{v}\right) d R_{v} \equiv 4 \pi<R_{v}>N_{v}, \\
k_{i v, i}^{2}=Z_{v, i}\left(\frac{4 \pi}{\Omega b}\right)^{1 / 2} \int_{x=2}^{\infty} x^{1 / 2} f_{i}(x)=2 \pi Z_{v, i} \int_{R_{i, \min }}^{\infty} R_{i} F_{i}\left(R_{i}\right) d R_{i} \equiv 2 \pi Z_{v, i}<R_{i}>N_{i},
\end{gathered}
$$

where

$$
<R_{v}>=\frac{\int_{R_{v, \text { min }}}^{\infty} R_{v} F_{v}\left(R_{v}\right) d R_{v}}{\int_{R_{v, \text { min }}}^{\infty} F_{v}\left(R_{v}\right) d R_{v}}, \quad<R_{v}>=\frac{\int_{R_{i, \text { min }}}^{\infty} R_{i} F_{i}\left(R_{i}\right) d R_{i}}{\int_{R_{i, \text { min }}}^{\infty} F_{i}\left(R_{i}\right) d R_{i}} .
$$

Although the integral of the SDFs shown in Eq. (7) has the same value for either phase space, the difference in dimensionality means the two SDF have quite different shapes in $\mathrm{r}$ - or $\mathrm{x}$ space. This is shown in Fig. 1, where $r$ - and $\mathrm{x}$-space SDFs calculated using the RT for a specific case are compared. Although use of the $\mathrm{x}$-space description may be more convenient for purposes of the calculations, the r-space SDF is more appropriate for comparing with experimental observations such as TEM measurements. 


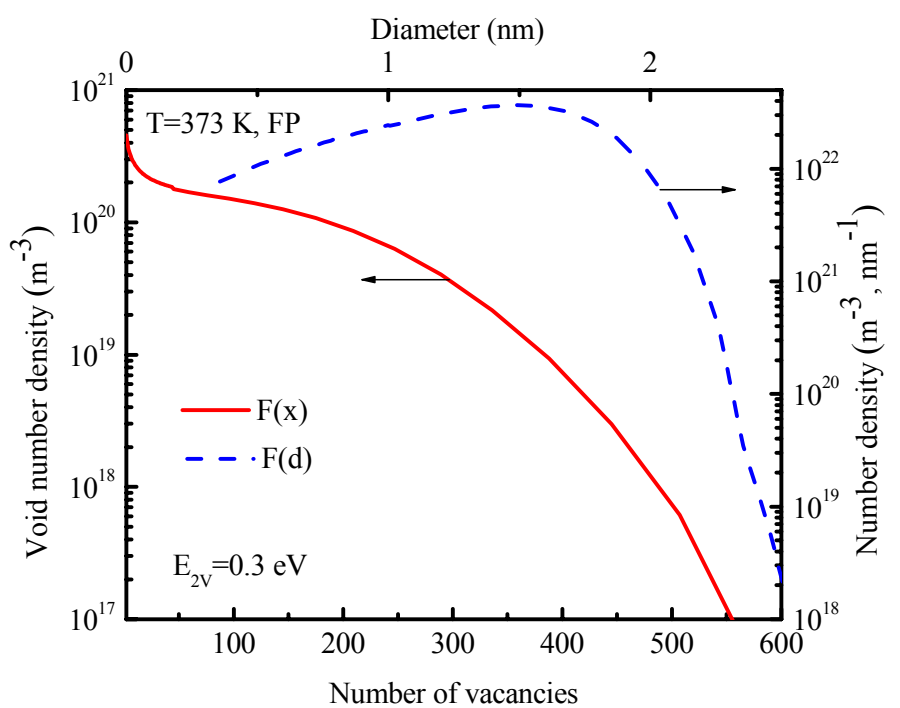

Fig. 1. Void size distribution function calculated for the case of $E_{2 v}=0.3: F(x)$ in phase space $x$ ( $x=$ number of vacancies), and $F(d)$ in phase space $d$ ( $d=$ void diameter).

\subsection{Initial and boundary conditions}

The initial and boundary conditions for SDFs of void, SIA loops and point defect concentrations are taken to have the following form

$$
\begin{gathered}
f_{v}(x, t=0)=C_{v 0} \delta(x-1), \quad f_{i}(x, t=0)=0, \quad(x \geq 1), \\
f_{v}(x=1, t)=C_{v}(t), \quad f_{i}(x=1, t)=C_{i}(t), \\
f_{v}(x=\infty, t)=f_{i}(x=\infty, t)=0, \\
C_{v}(t=0)=C_{v 0}, \quad C_{i}(t=0)=0,
\end{gathered}
$$

where $C_{v 0} \exp \left(-\frac{E_{v}^{f}}{k_{B} T}\right)$ is the thermal equilibrium vacancy concentration $\left(E_{v}^{f}\right.$ is the vacancy formation energy) and $\delta(x)$ is the Kronneker delta.

\subsection{Equations for point defect concentrations}

In order to complete the system of equations, we must add equations for the evolution of the vacancies, $C_{v}$, and SIAs, $C_{i}$, which are given by [3] 


$$
\begin{gathered}
\frac{d C_{v}(t)}{d t}=G_{N R T}\left(1-\varepsilon_{r}\right)\left(1-\varepsilon_{v}\right)-\left[\mu_{R} D_{i} C_{i}(t) C_{v}(t)+Z_{v}^{d} \rho D_{v}\left(C_{v}(t)-C_{v 0}\right)+\sum_{x=2}^{x=\infty} P_{i}(x) f_{i}(x, t)\right] \\
-\left[\left(P_{v}(1) f_{v}(1, t)-Q_{v}^{v}(2) f_{v}(2, t)\right)-P_{i}(2) f_{v}(2, t)\right]-\sum_{x=1}^{x=\infty}\left(P_{v}(x) f_{v}(x, t)-Q_{v}^{v}(x+1) f_{v}(x+1, t)\right) \\
\frac{d C_{i}(t)}{d t}=G_{N R T}\left(1-\varepsilon_{r}\right)\left(1-\varepsilon_{i}\right)-\left[\mu_{R} D_{i} C_{i}(t) C_{v}(t)+Z_{i}^{d} \rho D_{i} C_{i}+\sum_{x=1}^{x=\infty} P_{i}(x) f_{i}(x, t)\right] \\
-\left[P_{i}(1) f_{i}(1, t)-P_{v}(2) f_{i}(2, t)\right]-\sum_{x=1}^{x=\infty} Q_{v}^{i}(x+1) f_{v}(x+1, t)
\end{gathered}
$$

where $G_{N R T}$ is the point defect generation rate, $\mu_{R}$ is the recombination coefficient, $\rho$ is the dislocation density, and $Z_{v}^{d}, Z_{i}^{d}$ are the dislocation capture efficiencies for vacancies and SIAs, respectively (here $Z_{v}^{d}$ is set equal to 1.0 ). The first term on the right hand side of Eqs. (15) and (16) represents the generation rates of the mobile point defects, and the second term represents the capture rates by mutual point defect recombination, dislocations and the SIA loops. The third term accounts for the fact that reactions between two vacancies and two SIAs eliminate both of them at the same time, and the generation of vacancies and SIAs by reactions between a divacancy and SIA and di-SIA and vacancy. The last term represents the point defect capture rates by voids.

Eqs. (1)-(4) and(15), (16) are a set of coupled nonlinear equations and, in the general case, can be only solved by numerical methods. Eqs. (1) are systems of a large number of differential equations, which can be solved by numerical methods employing some grouping procedure in which the continuous distribution is represented as a histogram. In the present work the grouping method developed by Golubov, et al. [3] is used for the calculations.

Within the OKMC framework the same physical model simulated in a different way. Instead of assigning the values of the sink strengths for different defects, as in the framework of RT, the point defects are allowed to freely migrate in the simulation box of a given size filled with specified densities of defect sinks such as dislocations and point defect clusters distributed in the box. A brief description for the calculation techniques used in present work by the two methods is presented in the next section. 


\section{COMPUTATIONAL METHODS}

\subsection{The grouping method for approximate numerical solution of ME}

As shown in [3], there are two conservation laws which have to be satisfied within a grouping method in order to provide an accurate description, both the total number of clusters as well as the total number of defects contained in the clusters. A simple and accurate method to obtain such a description is to approximate the SDF within a given group clusters (specified by a range of sizes) by a linear function of the type [3]:

$$
f_{j}(x)=L_{0}^{j}\left(x-\langle x\rangle_{j}\right)+L_{1}^{j}
$$

The discrete cluster size classes are collected in groups of widths $\Delta x_{j}=x_{j}-x_{j-1}$ which include clusters of the sizes

$$
x_{j}=x_{j-1}+k,\left(k=1,2, \ldots \Delta x_{j}\right)
$$

where the subscript $j$ indicates the group number. Thus, each group consists of $n_{j}=\Delta x_{j}$ numbers of clusters of different sizes and is defined by the index " $j$ ". It can be shown that the mean cluster size within the $\mathrm{j}$-th group, $\langle x\rangle_{j}$, is equal to:

$$
<x>_{j}=x_{j}-\frac{1}{2}\left(\Delta x_{j}-1\right)
$$

Note that in a case when $\Delta x_{j}=1$ the mean size is equal to $\langle x\rangle_{j}=x_{j}$ which permits treating the case of $\Delta x_{j}=1$ the same way as other groups with $\Delta x_{j} \neq 1$. Note also that the first term in the right hand side of Eqs. (1) describing cascade cluster generation are normally non-zero only for the clusters of small sizes. In the following calculations, these smallest clusters are treated discretely, i.e. with $\Delta x_{j}=1$. Because of this fact, the grouping method presented below for $\Delta x_{j} \neq 1$ does not include these terms.

By appropriate manipulation of the set of kinetic equations of type Eq. (1), the following equations for the coefficients $L_{0}^{j}$ and $L_{1}^{j}$ can be obtained [3] 


$$
\begin{aligned}
& \frac{d L_{0}^{i}}{d t}=\frac{1}{\Delta x_{i}}\left[J\left(x_{i-1}\right)-J_{x}\left(x_{i}\right)\right], \\
& \frac{d L_{1}^{i}}{d t}=-\frac{1}{\Delta x_{i}}\left(\frac{\Delta x_{i}-1}{2 \sigma_{i}^{2}}\right)\left\{J_{x}\left(x_{i-1}\right)+J\left(x_{i}\right)-2 J\left(<x_{i}>-\frac{1}{2}\right)\right\},
\end{aligned}
$$

where $\sigma_{i}^{2}$ is the dispersion of cluster sizes in the group:

$$
\sigma_{i}^{2}=\frac{1}{\Delta x_{i}}\left[\sum_{\alpha=x_{i-1}+1}^{x_{i}} \alpha^{2}-\frac{1}{\Delta x_{i}}\left(\sum_{\alpha=x_{i-1}+1}^{x_{i}} \alpha\right)^{2}\right] .
$$

Eqs. (20) describe the evolution of the SDF within the group approximation. These equations satisfy the conservation laws obtained from the $\mathrm{ME}$ for the total number density of the clusters and the total number density of defects accumulated in the clusters. Note that Eqs. (20) are identical with Eqs. (1) in the limiting case when $\Delta x_{i}=1$. Because of this there is no problem in describing the SDF in the boundary region between the discrete and grouped clusters. Indeed, the first Eq. (20), which depends on $\Delta x_{i}$ only, transforms to Eqs. (1) for $\Delta x_{i}=1$, that is $f\left(x_{i}\right)=L_{0}^{i}$ and $L_{1}^{i}=0$. This is in contrast to the method suggested by Kiritani [1], where the equation describing the number density of the size class at the interface has a special form. Note that for the same group widths, this method requires twice as many equations as the Kiritani method but, as it was shown by calculations [3]. However, it has been shown to rather insensitive to the group widths; therefore, much wider (fewer) groups can be used. Note that due to a

typographical error the term $-\frac{1}{\Delta x_{i}}$ in the second Eq. (20) was omitted in Eq. (31) in [3] and the last term in brackets, $2 J\left(<x_{i}>-\frac{1}{2}\right)$, is equal to that given by Eq. (31) in [3] when the rates $P(x, t)$ and $Q(x, t)$ are taken to be constant within a group.

\subsection{OKMC computation method}

The general features of the OKMC code used in the present work, LAKIMOCA, have been described in [9]. Briefly, the model treats radiation produced defects (vacancies, self-interstitials atoms - SIA - and clusters thereof) as objects that have specific reaction volumes and that are located in particular positions in the simulation box. Each object can migrate and participate in a 
series of predefined reactions. The probabilities for physical transition mechanisms, which are basically migration jumps and emission from larger defects or from traps, are calculated in terms of Arrhenius frequencies for thermally activated events, $\Gamma_{i}=v_{i} \exp \left(-\frac{E_{a, i}}{k_{B} T}\right)$, where $v_{i}$ is the attempt frequency (pre-factor) for event $i$, and $E_{a, i}$ is the corresponding activation energy. The Monte Carlo algorithm [5] is used at each step to select the event that is going to take place, based on the corresponding probabilities. After a certain event is chosen, time is increased according to the residence time algorithm, $\Delta \tau=\left(\sum_{i=1}^{N_{e}^{\text {th }}} \Gamma_{i}+\sum_{j=1}^{N_{e}^{e x t}} P_{j}\right)^{-1}[10]$, where the $P_{j}$ are the probabilities of external events, such as the appearance of a cascade or of isolated Frenkel pair produced by impinging particles. The choice of this expression is in the long term equivalent to choosing $\Delta \tau^{\prime}=-\ln R^{*} \Delta \tau$, where $\mathrm{R}$ is a random number between 0 and 1 [11]. In addition, the model includes non-thermally activated events, such as the annihilation of a defect after encountering either a defect of opposite nature (i.e. a SIA encountering a vacancy) or a sink, as well aggregation, either by adding a point-defect to a cluster or by forming a complex between a defect and a trap for it. These events occur only on the basis of geometrical considerations (overlap of reaction volumes) and do not participate in defining the progression of time. It is possible to introduce different classes of immobile traps and sinks, characterized by specific geometrical shapes (spheres, infinite cylinders, surfaces etc.) suitable for mimicking voids and other defects such as dislocations and grain boundaries.

In the present work, the OKMC technique is used to simulate damage accumulation in the according to the framework of the model formulated above. A bcc iron lattice containing edge dislocations is simulated, with the evolution of two types of point defect clusters, voids (vacancy clusters) and SIA loops. To simplify the calculations, both types of defect clusters are treated as spherical absorbers. The only difference between the vacancy clusters and the SIA loops is that the first ones are described as neutral sinks, whereas the second are considered to be biased, i.e. having preference for absorption of SIAs. Thus, instead of using the second Eq.( 6), the sink strength of the SIA clusters is given by:

$$
k_{v, i}^{2}=Z_{v, i}^{l} w_{v} \sum_{x=2}^{\infty} x^{1 / 3} f_{i}(x) \equiv Z_{v, i}^{l} 4 \pi<R_{i}>N_{i}, \quad\left(Z_{v}^{l}=1, Z_{i}^{l}>1\right)
$$


The bias of the SIA clusters is equal to $p_{l}=\left(Z_{i}^{l}-Z_{v}^{l}\right) / Z_{v}^{l} \equiv Z_{i}^{l}-1$ and in the following calculations is chosen to be equal of that for the dislocations, i.e. $Z_{i}^{l}=Z_{i}^{d}$. Note that such an approach for the shape of SIA clusters in the following calculations is quite reasonable since the sink strengths of small spherical clusters and that of dislocation loops are similar when the cluster size is not large. Using Eqs. (6) one can find that the ratio of the sink strengths is given by

$$
\frac{\left(k_{i}^{2}\right)_{\text {sperical }}}{\left(k_{i}^{2}\right)_{\text {loop }}} \approx\left(\pi 3^{3 / 2}\right)^{1 / 6} x^{-1 / 6}=1.592 x^{-1 / 6},
$$

i.e. it only weakly depends on the number of defects in the cluster (it varies in a factor 2 when $x$ varies in a range from 2 to $10^{4}$ ). This size range matches well the calculations presented in this work since the highest irradiation dose used in the calculations is rather small $\left(10^{-2} \mathrm{dpa}\right)$.

The situation with simulation of dislocations is more complicated. One way of simulating this sink is direct introduction of an absorbing cylinder of a certain length, $L_{d}$, corresponding to the desired dislocation density, $\rho$, and with specified capture efficiencies for point defects. In this case, one may expect to reproduce the sink strength given in Eqs. (15) and (16) with the efficiencies $Z_{v, i}^{d}=\frac{2 \pi}{\ln \left[\left(k R_{c v, c i}\right)^{-1}\right]}$, which leads to a dislocation bias to be equal to:

$$
p=\frac{Z_{i}^{d}-Z_{v}^{d}}{Z_{v}^{d}}=\frac{\ln \left(R_{d i} / R_{d v}\right)}{\ln \left[\left(k R_{d i}\right)^{-1}\right]}
$$

where $r_{d i}, r_{d v}$ are the cylinder capture radii for SIAs and vacancies, respectively, and $k=\sqrt{k^{2}}$ (where $k^{2}$ is the total sink strength in the crystal). This approach looks quite natural and simple, but in fact this is not the case. The first problem arises with choosing the cylinder length. In order to simulate a given dislocation density, $\rho$, the length of the cylinder has to be taken to be equal to $L_{d}=\rho V_{b o x}$, which may be too small or too large to fit conveniently in the simulation box, $L_{b o x}=V_{b o x}^{1 / 3}$. In addition, $\rho$ can vary by several orders of magnitude whereas the maximum box size is essentially fixed by computational limitations. As a result, such a cylinder will have its ends inside the simulation box; thus it cannot reproduce the actual cylindrical symmetry of a dislocation. The impact of this on the dislocation sink strength requires further investigation, 
which is beyond the scope of the present work, and is not directly relevant to the comparison of the RT and OKMC methods. A second issue arises from the fact that the dislocation sink strength and corresponding bias depends on the total sink strength, see Eq. (24), $k^{2}$, which changes during the irradiation. Accounting for this would lead to an increase of the number of variable parameters that may complicate the comparison.

A way to avoid these complications is to simulate the dislocation sink by introducing another kind of spherical absorbers, which maintain the two main properties of dislocations as a sink for point defects: (a) a preference for SIA absorption, i.e. maintain a constant dislocation bias, and (b) maintain constant sink strength during irradiation. This may be achieved by introducing additional spherical absorbers with invariable (during irradiation) density and size as follows

$$
\begin{gathered}
k_{v d}^{2}=4 \pi R_{v}^{d} N^{d} \equiv \rho, \\
k_{i d}^{2}=4 \pi R_{i}^{d} N^{d}, \quad\left(R_{i}^{d}>R_{v}^{d}\right) .
\end{gathered}
$$

Thus choosing a specific capture radius for vacancies, $R_{v}^{d}$, the density of the absorbers, $N^{d}$, can be calculated from the first Eq. (25), $N^{d}=\frac{\rho}{4 \pi R_{v}^{d}}$. The bias in the case is equal to

$$
p=\frac{k_{i}^{2}-k_{v}^{2}}{k_{v}^{2}}=\frac{R_{i}^{d}}{R_{v}^{d}}-1,
$$

i.e. it depends on the ratio of $R_{i}^{d} / R_{i}^{d}$ only. Taking the ratio $R_{i}^{d} / R_{i}^{d}$ to be equal to $Z_{i}^{d}$ one may expect that Eqs. (25) will reproduce the sink strength of dislocations with respect to their ability to capture point defects. Thus, Eqs. (25) and (26) permit calculating the absorber properties of dislocation at any given dislocation density and bias.

A box of $300 \times 300 \times 300$ lattice parameters was used in all the simulations discussed here. Given the lattice parameter of $\alpha$-Fe (see Table 2), this leads to a volume equal to $6.423 \times 10^{-22} \mathrm{~m}^{-3}$. When the spherical absorber approximation is used for the dislocations, the "dislocation" absorbers with capture radii of $R_{v}^{d}=0.4 \mathrm{~nm}, R_{i}^{d}=0.48 \mathrm{~nm}$ for vacancies and SIAs, respectively, are randomly distributed in the simulation box. If the cylinder approximation is used for dislocations, a cylinder of a given length is introduced in the middle of the simulation box along a cubic axis. Irradiation is simulated by introducing isolated point defects (Frenkel pair 
production), or, for simulating cascade damage production, together with small vacancy or SIA clusters that are introduced in randomly selected positions within the simulation volume.

Essentially three types of irradiation are considered:

1. Pure Frenkel pair (FP) production,

2. Defect generation by cascades (type I): $35 \%$ of vacancies produced in a form of nanovoids), or

3. Defect generation by cascades (type II): $35 \%$ of SIAs produced in a form of nano- spherical clusters)

The cascade efficiency, i.e. the fraction of point defects that survive after cascade cooling phase, is taken to be the same in all three cases.

The binding energy of a vacancy with a void of size $x$ is described using the capillarity model adjusted to a specific di-vacancy binding energy, $E_{2 v}$. The general expression for the vacancy binding energy takes the following form:

$$
E_{v}^{b}(x)=E_{v}^{f}+\left(E_{2 v}-E_{v}^{f}\right)\left(\frac{x^{2 / 3}-(x-1)^{2 / 3}}{2^{2 / 3}-1}\right) .
$$

The energy $E_{2 v}$ is treated as a variable parameter to permit investigation of the stability of the vacancy clusters. The SIA clusters are taken to be thermally stable. The material and irradiation parameters are given in Table 2 . 
Table 2. Material and irradiation parameters used in calculations

\begin{tabular}{|l|l|}
\hline Temperature, $\mathrm{T}$ & $373,523 \mathrm{~K}$ \\
\hline Lattice parameter, $a$ & $0.2876 \mathrm{~nm}$ \\
\hline Atomic volume, $\Omega=a^{3} / 2$ & $1.189 \times 10^{-29} \mathrm{~m}^{3}$ \\
\hline Number of atoms in unit volume, $N=1 / \Omega$ & $8.407 \times 10^{28} \mathrm{~m}^{-3}$ \\
\hline Box volume of a cube of size $300 \mathrm{a}, V_{b o x}$ & $6.423 \times 10^{-22} \mathrm{~m}^{3}$ \\
\hline Number of atoms in the box, $N_{b o x}=V_{b o x} / \Omega$ & $5.4 \times 10^{7}$ \\
\hline Number density equivalent to one cluster in, $N_{c l}=1 / V_{b o x}$ & $1.557 \times 10^{21} \mathrm{~m}^{-3}$ \\
\hline NRT displacement rate, $G_{N R T}$ & $10^{-6} \mathrm{dpa} / \mathrm{s}$ \\
\hline Fraction of point defect recombined in cascades, $\varepsilon_{r}$ & 0.60 \\
\hline Fraction of SIAs in cluster form, $\varepsilon_{i}(\mathrm{x}=2,3$ and 4$)$ & 0.35 \\
\hline Fraction of voids in cluster form, $\varepsilon_{v}(\mathrm{x}=6)$ & 0.35 \\
\hline Effective displacement rates, $G_{v}=G_{i}=G_{N R T}\left(1-\varepsilon_{r}\right)$ & $4.0 \times 10^{-7} \mathrm{dpa} / \mathrm{s}$ \\
\hline Recombination coefficient, $\mu_{R}=4 \pi\left(r_{v}+r_{i}\right) / \Omega,\left(r_{v}+r_{i}=0.4466 \mathrm{~nm}\right)$ & $4.72 \times 10^{20} \mathrm{~m}^{-2}$ \\
\hline Attempt frequency, $v$ & $6.0 \times 10^{12} \mathrm{~s}^{-1}$ \\
\hline Vacancy diffusion coefficient, $D_{v}=D_{v 0} \exp \left(-\frac{E_{v}^{m}}{k T}\right)$ & \\
\hline pre-exponential, $D_{v 0}=\frac{1}{6} r^{2} v,\left(r=\frac{\sqrt{3}}{2} a\right)$ & $6.02 \times 10^{-8} \mathrm{~m}^{2} / \mathrm{s}$ \\
\hline migration energy, $E_{v}^{m}$ & $0.65 \mathrm{eV}$ \\
\hline SIA diffusion coefficient, $D_{i}=D_{i 0} \exp \left(-\frac{E_{i}^{m}}{k T}\right)$ & $0.30 \mathrm{eV}$ \\
\hline pre-exponential, $D_{i 0}=\frac{1}{6} r^{2} v$ & $0.2-0.8 \mathrm{eV}$ \\
\hline Binding energy of di-vacancies, $E_{2 v}$ & $1.0,1.20$ \\
\hline Capture efficiencies of dislocations and SIA clusters, $Z_{v}^{l}, Z_{i}^{l}$ & $10^{14} \mathrm{~m}^{-2}$ \\
\hline Dislocation density, $\rho_{d}$ & $0.48 \mathrm{and} 0.4 \mathrm{~nm}$ \\
\hline Capture radii for “dislocation" spherical absorbers, $R_{i}^{d}, R_{v}^{d}$ & $6.02 \times 10^{-8} \mathrm{~m}^{2} / \mathrm{s}$ \\
\hline
\end{tabular}




\section{RESULTS OF CALCULATIONS}

If point defects and defect clusters generated by irradiation appear randomly in space and uniform in time, the RT approach is fully applicable, i.e. the cluster evolution in this case can be rationalized on the basis of the results obtained from the RT calculations. Thus, in order to characterize the main features of point defect cluster evolution, we will first present the results obtained from the RT method at the specified irradiation conditions for the case of FP and then will make a comparison the RT results and those obtained from the OKMC simulation for the same irradiation conditions.

\subsection{Cluster evolution at Frenkel pair production at $373 \mathrm{~K}$}

The simplest type of irradiation, production of only Frenkel pair, will be analyzed first. This approximately corresponds to damage production under high energy electron irradiation. Since only homogeneous nucleation of point defect clusters is possible in this case, void nucleation is very sensitive to the cluster stability, see Eq. (27). Recall that SIA clusters are considered to be thermally stable. The binding energy of a vacancy to voids calculated using Eq. (27) for different values of $E_{2 v}$ in the range of 0.2 to $0.8 \mathrm{eV}$ is presented in Fig. 2. The binding energy increases quickly with cluster size and increases at a given size for larger values of $E_{2 v}$. One would therefore expect that void evolution will proceed in such a way that the void density will increase with increasing of $E_{2 v}$.

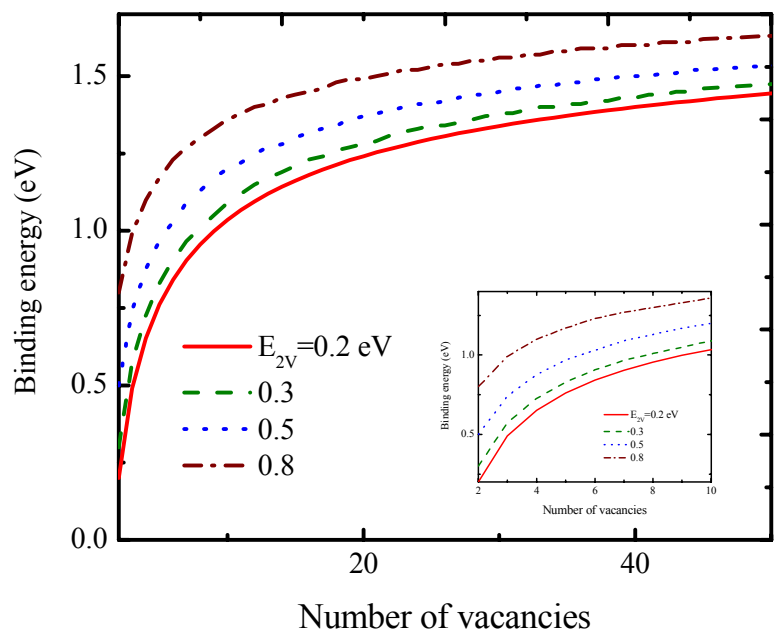

Fig. 2. The binding energy of vacancies with voids at different magnitude of the binding energy of di-vacancy, $E_{2 v}$, calculated with use of Eq. (27). 
The dose dependence of the total number density of voids and SIA clusters calculated for different energies $E_{2 v}$ is presented in Fig. 3. The calculations indicate that the void density at $100^{\circ} \mathrm{C}$ is essentially insensitive to $E_{2 v}$ for values greater than $0.5 \mathrm{eV}$, i.e. for $E_{2 v}=0.5 \mathrm{eV}$ void stability at this temperature is high enough that vacancy emission does not play any role. It can be seen that the void density is much higher than that of the density of SIA clusters in all cases. Such a difference may be understood by taking into account that the homogeneous cluster nucleation is also very sensitive to the point defect concentrations. Since the vacancy diffusivity is much lower than that of SIAs, the steady state vacancy concentration is much higher than that of the steady state SIA concentration as can be seen in Fig. 4 where the point defect densities calculated for three different di-vacancy binding energies are presented. As can be seen from Fig. 4, the point defect concentrations obey a typical behavior: the SIA concentration reaches steady state by a dose of about $10^{-9} \mathrm{dpa}$, whereas the steady state vacancy concentration reaches a much higher value at much higher dose (about $10^{-5} \mathrm{dpa}$ ). The slow change in point defect concentrations at doses higher than $\sim 10^{-5}$ dpa reflects the impact of growing voids on the total sink strength. This increasing sink strength slowly reduces the point defect concentrations.

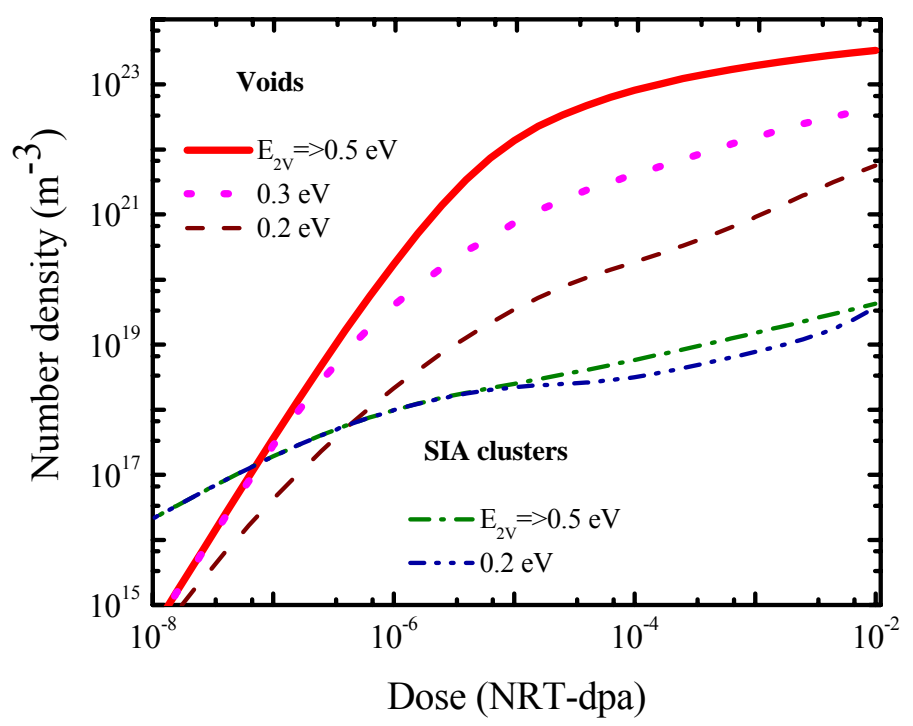

Fig. 3. Dose dependence of the total number density of voids and SIA clusters calculated at $373 \mathrm{~K}$ for different di-vacancy binding energies. Note that the SIA cluster density is much low than that of the voids and practically insensitive the void energetic. 


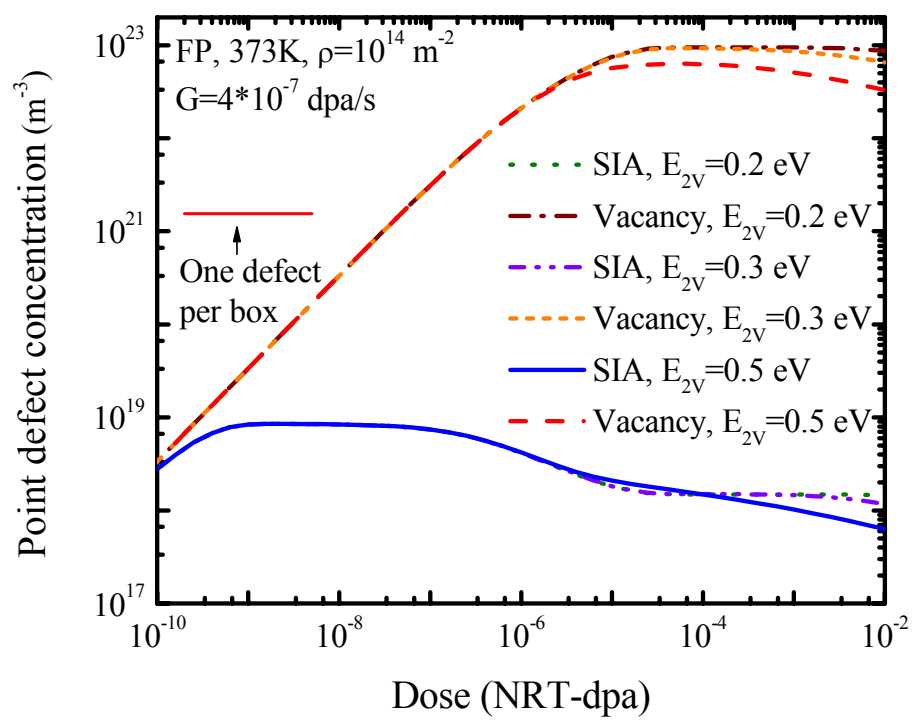

Fig. 4. Dose dependence of point defect concentration calculated at $373 \mathrm{~K}$ for different divacancy binding energies. Note that the short red line marks the concentration which corresponds to a case when one defect only placed in the OKMC simulation box.

The short red line on Fig. 4 marks the concentration which corresponds to the case when one point defect is placed in the OKMC simulation box. The "one defect" concentration is much higher than the steady state SIA concentration. Thus, one may conclude that it may be difficult or even impossible to compare the RT and OKMC results for the SIA concentration for the specified box size. The steady state vacancy concentration on the other hand is high enough for such a comparison at these conditions. However, at higher temperatures or lower displacement rates, the same problem may arise for the vacancy concentration also. This is illustrated in Fig. 5 where the calculated dose dependence for point defect concentrations is presented for three different temperatures. As shown in Fig. 5, the vacancy concentration at $523 \mathrm{~K}$ is smaller than that which corresponds to the case of only one defect in the OKMC simulation box. This implies that $\mathrm{OKMC}$ simulations at temperatures of $523 \mathrm{~K}$ and higher for a point defect generation rate of about $10^{-6} \mathrm{dpa} / \mathrm{s}$ may not be capable of accurately reproducing point defect evolution. The impact of this issue can be demonstrated by systematic comparisons of damage accumulation calculated by the two simulation techniques for different irradiation conditions. 


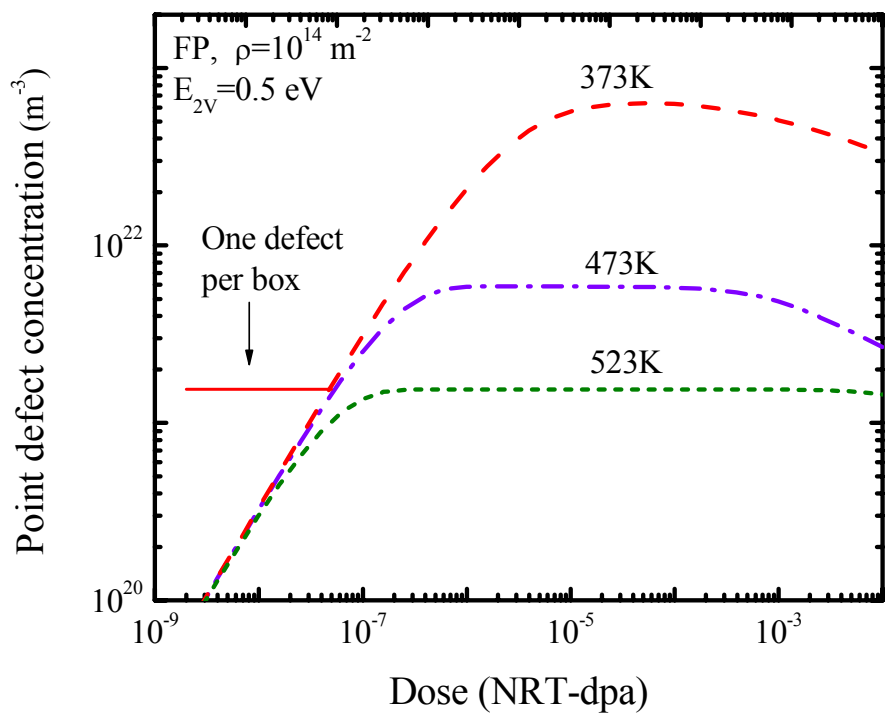

Fig. 5. Dose dependence of vacancy concentration calculated at different temperatures for the case $E_{2 v}=0.5 \mathrm{eV}$. Note that the vacancy concentration at $523 \mathrm{~K}$ is already below of that which corresponds to a case when one defect only placed in the OKMC simulation box.

To complete the description of the results obtained with the RT method, the SDFs calculated for the different vacancy binding energies, and the corresponding dose dependence of swelling and the void sink strength will be discussed.
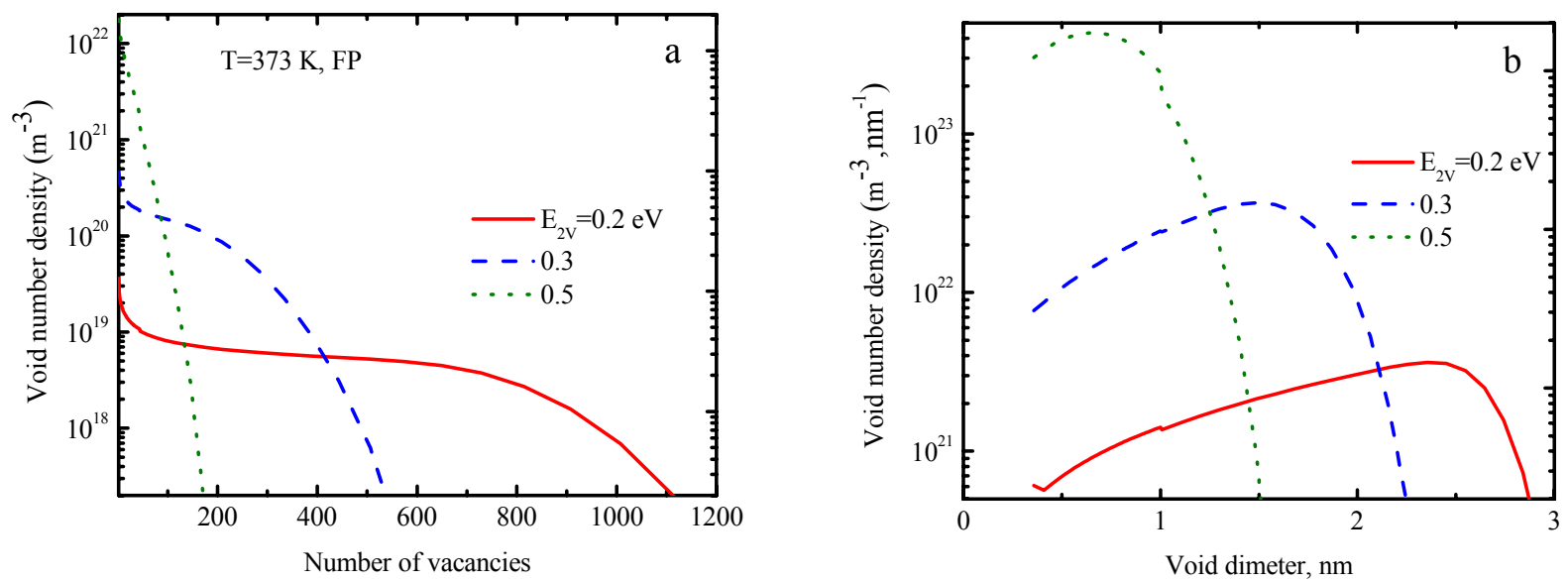

Fig. 6. Size distribution function of voids calculated for the cases of $E_{2 v}=0.2,0.3$ and $0.5 \mathrm{eV}$ : functions $F(x)$ in (a) and corresponding functions $F(r)$ in (b). Note that the SDFs in $x$ - and $r$ space have quite different shape, scale and dimensionality.

Void size distribution functions calculated for the cases of $E_{2 v}=0.2,0.3$ and $0.5 \mathrm{eV}$ are shown in Fig. 6. The functions $F(x)$ are shown in Fig 6(a) and the corresponding functions $F(r)$ 
in Fig. 6(b). As mentioned above, the SDFs have quite different shape, scale and dimensionality in $x$ and $r$ phase space. The vacancy cluster SDF shown in Fig. 6 follows the expected behavior; an increase in void stability results in higher density and smaller mean size.

The dose dependence of swelling and the sink strength of voids calculated for the cases of $E_{2 v}=0.2,0.3$ and $0.5 \mathrm{eV}$ are presented in Fig. $7 \mathrm{a}$ and $7 \mathrm{~b}$, respectively. The plots demonstrate that total vacancy accumulation increases with an increase in void stability. The main reason for this is the faster increase in the sink strength in the case of more stable voids which permits the void sink strength to reach values close to that of the dislocation sink strength, which normally results in a higher swelling rate [12]. This is clearly seen in Fig. 8 where the dose dependence of the swelling rate corresponding to the swelling shown in Fig. 7a is presented (see the thick curves). The swelling rate is of orders of magnitude larger in the case of the stable voids compared to that of less stable ones. Moreover the swelling rate in the case of stable voids differs qualitatively from that of unstable ones, showing non-monotonic behavior with a maximum value larger than that which may be expected from analytical considerations (see below). In order to demonstrate the reason for this difference in swelling rate in the case when $K_{v}(x)=0$, one can integrate the product of the first ME (Eq. 1(a)) multiplied by $x$, in order to get an equation for the swelling rate, $\frac{d S(t)}{d t}[3]$ :

$$
\frac{d S(t)}{d t} \equiv \sum_{x=2}^{\infty} x \frac{\partial f_{v}(x, t)}{\partial t}=J_{v}(1, t)+\sum_{x=2}^{\infty} J_{v}(x, t) .
$$
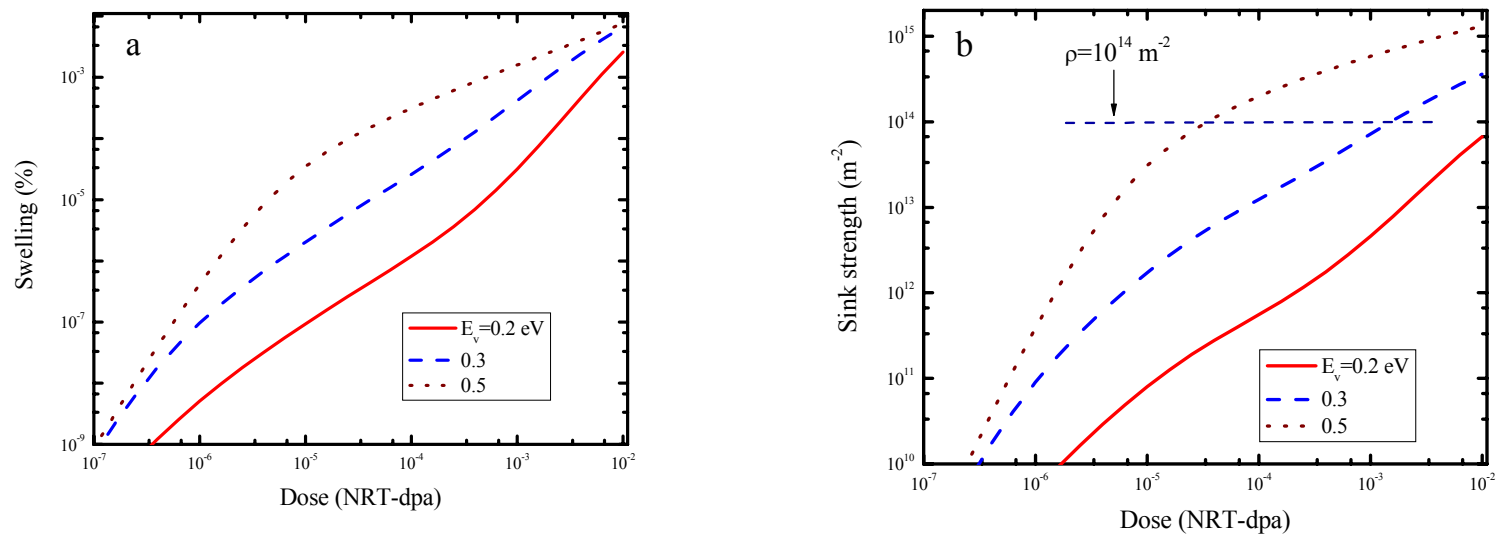

Fig. 7. Dose dependence of swelling, a, and the sink strength of voids, $b$, calculated for the cases of $E_{2 v}=0.2,0.3$ and $0.5 \mathrm{eV}$. Note that the thin dash line corresponds to the sink strength of dislocations. 
Neglecting thermal emission of vacancies from the voids, which does not play any significant role in vacancy accumulation, Eq. (28) may be presented in the following form:

$$
\frac{d S(t)}{d t}=\left[2 P_{v}(1, t) C_{v}(t)-Q_{v}^{i}(2, t) f(2, t)\right]+\sum_{x=2}^{\infty}\left[P_{v}(x, t)-Q_{v}^{i}(x, t)\right] f(x, t) .
$$

Taking into account Eqs. (3) and (6), the swelling rate may be finally presented in the following form:

$$
\frac{d S(t)}{d t}=\left[2 P_{v}(1, t) C_{v}(t)-Q_{v}^{i}(2, t) f(2, t)\right]+\left[D_{v} C_{v}-D_{i} C_{i}\right] k_{v}^{2}
$$

The first term on the right hand side of Eq. (30) represents impact of void nucleation, i.e. production of di-vacancies, whereas the second one represents vacancy accumulation in voids of all sizes. Because void nucleation decreases as the irradiation dose increases, the second term on the right hand side of Eq. (30) becomes dominant. This can be used to check validity of the calculated results obtained via any numerical technique. However, the direct use of Eq. (30), which may be easily done in the framework of RT, may be difficult in the case of OKMC. The reason is that the steady state point defect concentrations, particularly the SIA concentration, $C_{i}$, may be much lower than can be calculated in a general case (see Fig. 4). However, taking into account that the point defect concentrations reach steady state when the void density reaches saturation as given by [14]:

$$
\begin{aligned}
& D_{v} C_{v}=\frac{2 G}{\left(k_{v}^{2}+Z_{v}^{d} \rho\right)}\left(1+\sqrt{1+\frac{4 G \mu}{D_{v}\left(k_{v}^{2}+Z_{v}^{d} \rho\right)\left(k_{v}^{2}+Z_{i}^{d} \rho\right)}}\right)^{-1}, \\
& D_{i} C_{i}=D_{v} C_{v} \frac{\left(k_{v}^{2}+Z_{v}^{d} \rho\right)}{\left(k_{v}^{2}+Z_{i}^{d} \rho\right)}
\end{aligned}
$$

where $G$ is the rate of stable Frenkel pair generation. Combining Eqs. (30) with Eq. (31), the swelling rate at a high enough dose, i.e. beyond the nucleation stage, can be written as:

$$
\frac{d S}{d\left(G_{N R T} t\right)}=p_{d} \frac{k_{v}^{2} Z_{v}^{d} \rho}{\left(k_{v}^{2}+Z_{i}^{d} \rho\right)} \frac{2\left(1-\varepsilon_{r}\right)}{\left(k_{v}^{2}+Z_{v}^{d} \rho\right)}\left(1+\sqrt{1+\frac{4 G_{N R T}\left(1-\varepsilon_{r}\right) \mu_{R}}{D_{v}\left(k_{v}^{2}+Z_{v}^{d} \rho\right)\left(k_{v}^{2}+Z_{i}^{d} \rho\right)}}\right)^{-1},
$$


an expression which can be easily used by OKMC since the sink strength of the spherical absorbers is reproduced by the technique with very high accuracy [9, 13]. Note that Eq. (32) can be easily generalized for the case when cascade generation of voids and SIA clusters is taken into account and the corresponding in-cascade clustering fractions are equal to each other, i.e. at $\varepsilon_{v}=\varepsilon_{i}$. In this case, the swelling rate consists of two parts: accumulation via freely migrating point defects, and accumulation via cascade clustering (see Eq. (1a). The swelling rate is then given by:

$$
\begin{aligned}
\frac{d S}{d\left(G_{N R T} t\right)}= & \left(1-\varepsilon_{v}\right) p_{d} \frac{k_{v}^{2} Z_{v}^{d} \rho}{\left(k_{v}^{2}+Z_{i}^{d} \rho\right)} \frac{2\left(1-\varepsilon_{r}\right)}{\left(k_{v}^{2}+Z_{v}^{d} \rho\right)}\left(1+\sqrt{1+\frac{4 G_{N R T}\left(1-\varepsilon_{r}\right) \mu_{R}}{D_{v}\left(k_{v}^{2}+Z_{v}^{d} \rho\right)\left(k_{v}^{2}+Z_{i}^{d} \rho\right)}}\right)^{-1} \\
& +\left(1-\varepsilon_{r}\right) \varepsilon_{v}\left[1-\frac{k_{v}^{2}}{\left(k_{v}^{2}+Z_{i}^{d} \rho\right)}\right] .
\end{aligned}
$$

The multiplier $\left(1-\varepsilon_{v}\right)$ in the first term of Eq. (33) accounts for the reduction in production of freely migrating vacancies and SIAs. The negative term in the square bracket in the second term accounts for reactions of voids with that fraction of SIAs which is not subject to the mutual recombination with vacancies.
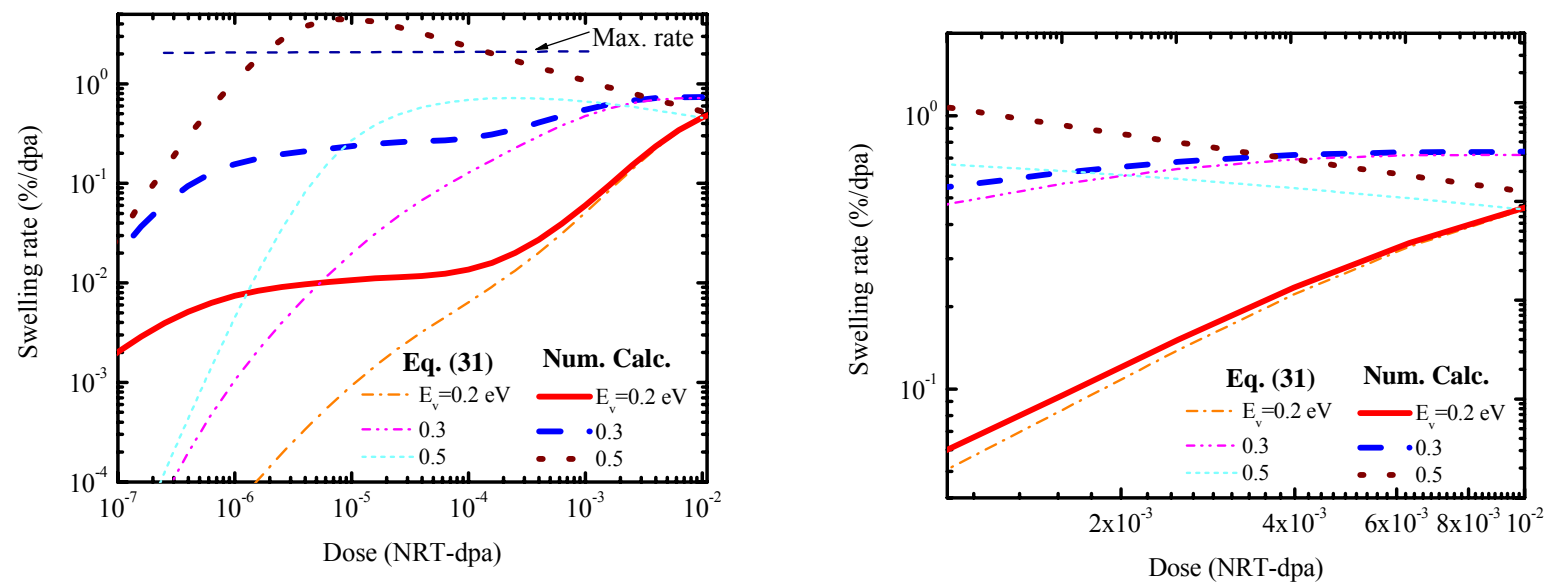

Fig. 8. Dose dependence of swelling rate corresponding to swelling presented in Fig. 7a by the thick curves. Note that the two plots are equivalent - the difference is the scaling of the axes.

The thin curves in Fig. 8 represent the results of calculations based on Eq. (32) with the sink strengths presented on Fig. 7b. As can be seen from Fig. 8, the swelling rate at low doses is much larger than that described by Eq. (32). This demonstrates the impact of void nucleation on 
vacancy accumulation. Note that in the case of stable voids, $E_{2 v}=0.5 \mathrm{eV}$, the instantaneous swelling rate is even larger than the maximum swelling rate obtained from Eq. (32). However, at large enough doses void nucleation does not play an essential role and the swelling rate is fully determined by the total sink strength of voids and dislocations. At this point, the numerical results coincide with those given by Eq. (32). This indicates that the numerical results obtained from the RT using the grouping method presented above are fully consistent with the analytical analysis.

\subsection{Comparison of results obtained by use of RT and OKMC methods.}

4.2.1 Cluster evolution for Frenkel pair production only and with dislocation bias equal to zero. Although it is strictly unrealistic case, the case of zero bias is considered to test both methods' ability to reproduce cluster evolution when point defect accumulation can only take place due to homogeneous cluster nucleation. Generally speaking, the point defect concentration should reach a quasi-steady-state when the cluster densities reach terminal values. Thus, the results are very sensitive to the accuracy of calculated reaction rates of point defect defects with each other, and with different sinks.

The dose dependence of point defect concentrations obtained by the two methods is presented in Fig. 9a. The vacancy concentration obtained by OKMC is slightly higher than that obtained by RT but the difference is not large and decreases with dose. In contrast the SIA concentration calculated by RT is almost two orders of magnitude smaller than that obtained by OKMC. The reason for this is obvious since the OKMC SIA density is practically equal to that of one defect per simulation box, i.e. the minimal density achievable at the given box size. Thus one may conclude that the SIA concentration is rather artificial in the case of OKMC and, consequently no valid comparison of the SIA concentrations can be made. 

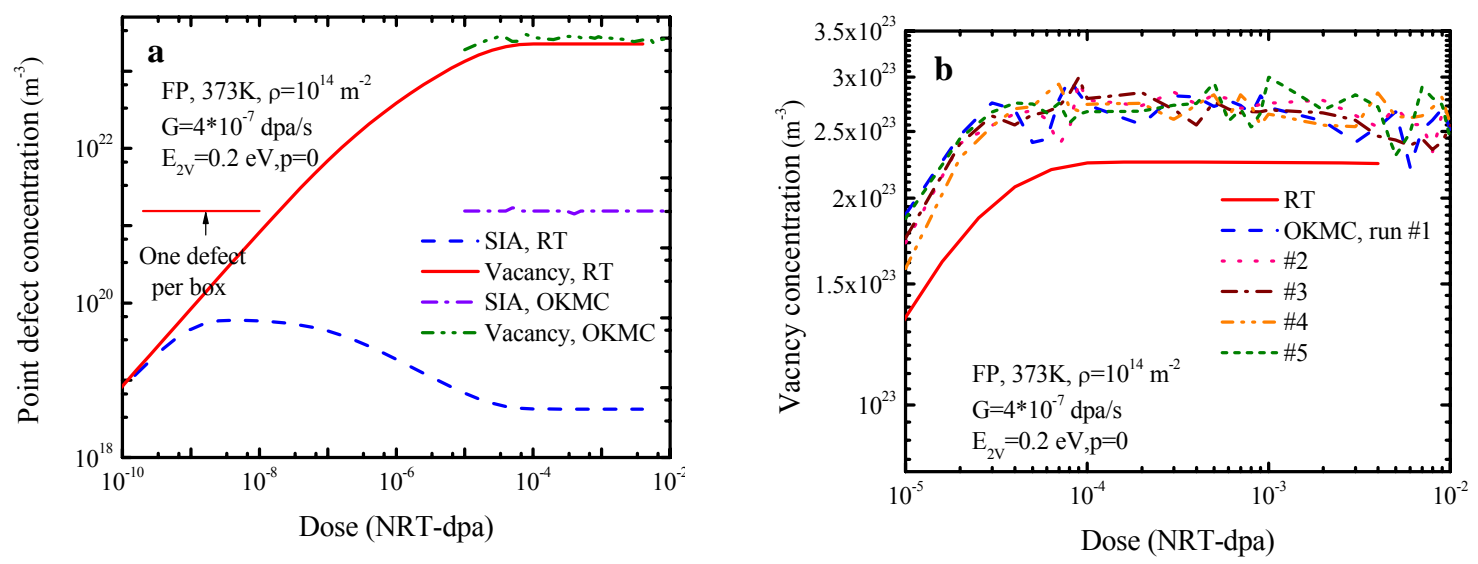

Fig. 9. Dose dependence of point defect concentrations calculated for the case of Frenkel pair production with $p=0$ using $\mathrm{RT}$ and OKMC methods (a). In part (b) the dose dependence of vacancy concentration is presented with results obtained by OKMC from 5 runs which differ in the initial spatial distribution of the dislocation sinks.

The dose dependence of cluster density obtained by the two methods is presented in Fig. 10a. The void density obtained by RT is substantially smaller than that obtained by OKMC. Formally speaking the same result can be seen for the SIA clusters. However it will be more correct to conclude that there are no SIA clusters at all in the case of OKMC since only one SIA cluster was found at a single dose. Thus one may conclude that the OKMC method is not relevant to analyze SIA cluster formation at these irradiation conditions.
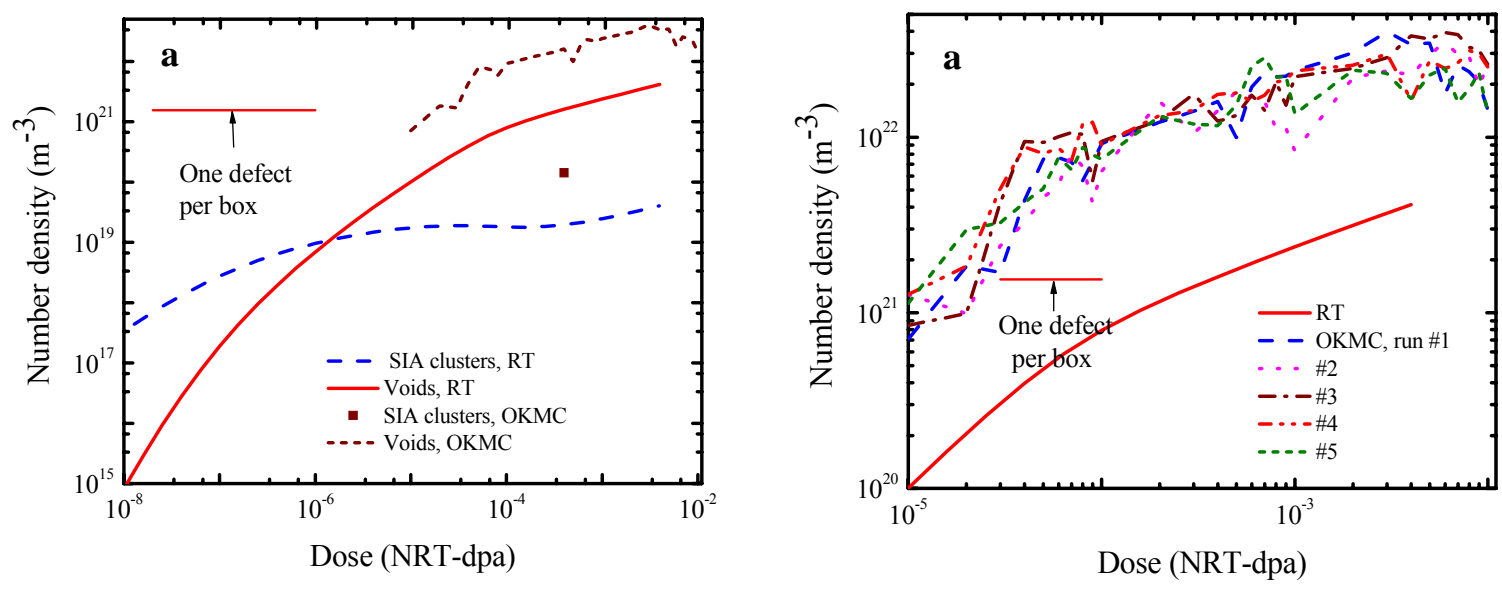

Fig. 10. Dose dependence of point defect cluster number density calculated for the case of Frenkel pair production with $p=0$ using RT and OKMC methods (a). In part (b), only the dose dependence of voids is presented with results obtained by OKMC for 5 runs which differ in the initial spatial distribution of the dislocation sink. 
The comparison of the dose dependence of vacancy concentration and the void number density obtained by the RT calculations and those obtained by OKMC from 5 runs for the case of $p=0$, which differ from each other by the initial spatial distribution of the "dislocation sink" simulated by the spherical absorbers is presented on Fig. $9 \mathrm{~b}$ and $10 \mathrm{~b}$. The figures provide an indication of the statistical variations in the OKMC results for the void density and vacancy concentration. The results span a significant range, but the values all fall higher than that obtained by RT. The reason for such difference is not clear and will be studded later. It is worth to noting that the $\mathrm{OKMC}$ results are self-consistent: the higher vacancy concentration leads to the nucleation of a higher density of vacancy clusters compared to that of the RT results.

Void size distribution functions calculated by the two methods are presented in Fig. 11. There are substantial differences between the two: (a) the SDF obtained by OKMC is not continuous in contrast to the one obtained by RT, (b) the void density at small sizes obtained from OKMC is almost one order of magnitude larger that that from the RT, and (c) there is a group of voids in the OKMC that are much larger than those obtained from the RT calculations. As a result the total number of vacancies accumulated in voids calculated by OKMC is almost one order of magnitude higher than that calculated by RT.

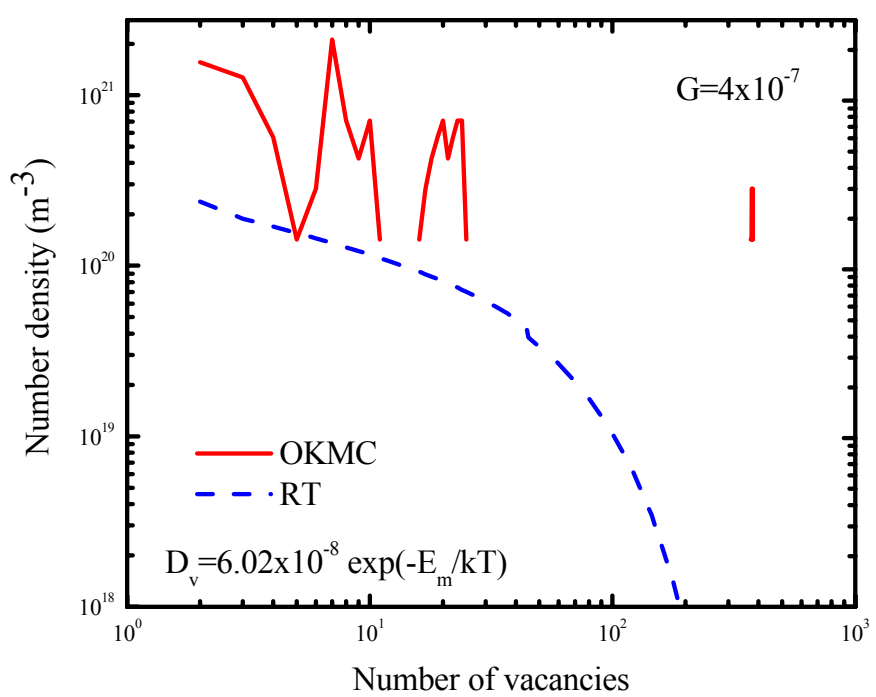

Fig. 11. Size distribution functions of voids at $0.01 \mathrm{dpa}$ calculated by the two methods for the case of $E_{2 v}=0.2$ and $p=0$. 
4.2.2 Cluster evolution with Frenkel pair production only and a dislocation bias of $20 \%$.

The dose dependence of point defect concentrations obtained by the two methods in the case of $p=20 \%$ are presented in Fig. 12. The vacancy concentrations obtained by both methods are close to each other, similar to that in the case of $p=0$. The situation with the SIA concentration is also the same as described above. Thus, one may conclude that OKMC and RT provide very similar values of the vacancy concentration regardless of the magnitude of the dislocation bias at these irradiation conditions and simulation box size.

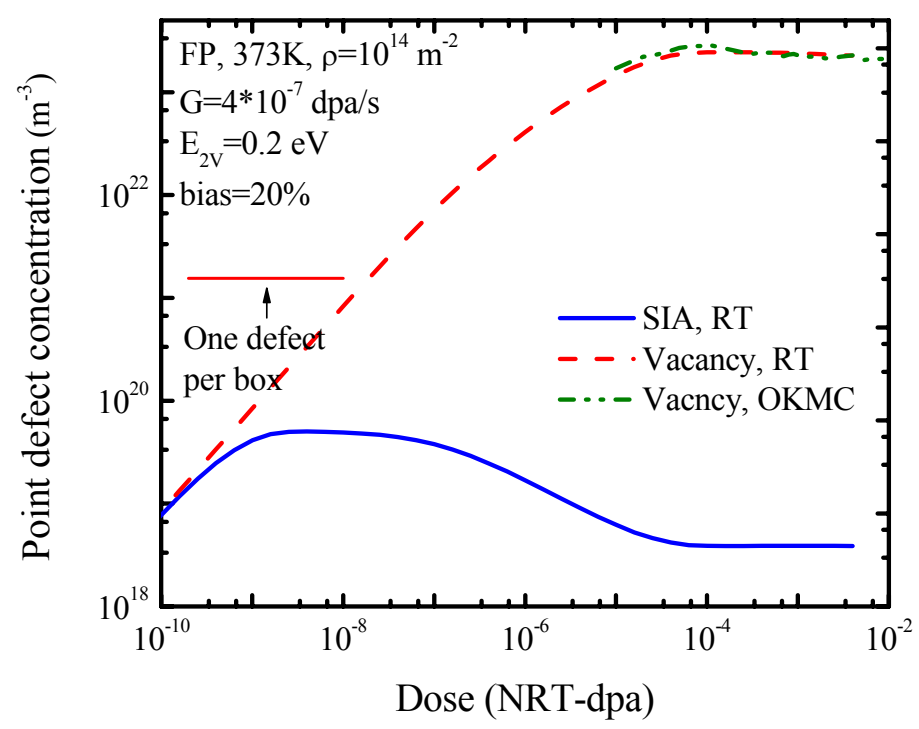

Fig. 12. Dose dependence of point defect concentrations calculated for the case of Frenkel pair production with $p=20 \%$ using RT and OKMC methods.

Size distribution functions of voids calculated by the two methods for the case of $p=20 \%$ are presented in Fig. 13. The differences between the results obtained from the two methods are very similar to that obtained for the case $p=0$. The SDF obtained by OKMC is not continuous, the void density from OKMC at small sizes is almost one order of magnitude larger than that from RT, and there is a group of voids in the OKMC simulation which are much larger than those from the RT calculations. As a result, the total number of vacancies accumulated in voids calculated by $\mathrm{OKMC}$ in the case of non-zero bias is almost one order of magnitude higher than that calculated by RT. The total number density of voids calculated by OKMC in this case is also much larger than those calculated by RT. 


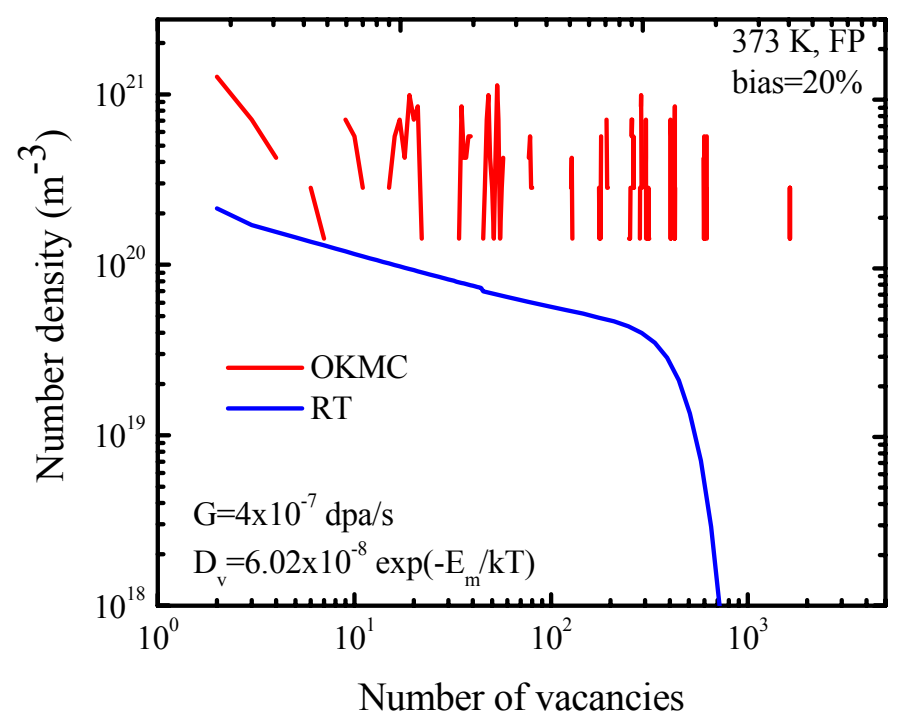

Fig. 13. Size distribution functions of voids at 0.01 dpa calculated by the two methods for the case of $E_{2 v}=0.2$ and $p=20 \%$.

4.2.3. Cluster evolution with in-cascade generation of voids and a dislocation bias of $20 \%$.

The total point defect production rate is the same in this case, but the production rate of single vacancies is reduced by $35 \%$. The corresponding number of vacancies is introduced into the simulation box in the form of di-vacancies and tri-vacancies. The dose dependence of point defect concentrations obtained by the two methods in this case is presented in Fig. 14. The vacancy concentration obtained by OKMC is somewhat larger than that obtained by RT at low doses and lower at higher doses. The situation with the SIA concentration is the same as described above. The agreement between the values of the vacancy concentration calculated by the two methods in this case is not as good as in the case of only FP production. This difference may be partially explained by the statistical variation in the OKMC results shown in Fig. 9(b). 


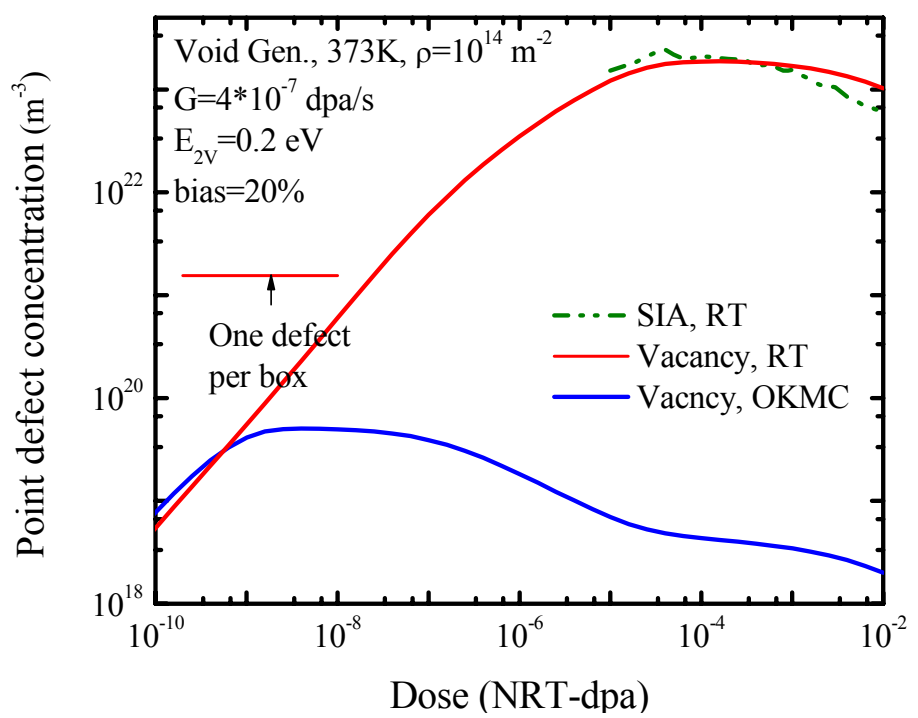

Fig. 14. Dose dependence of point defect concentrations calculated for the case of cascade generation of voids with $p=20 \%$ using RT and OKMC methods.

The voids size distribution function calculated by the two methods for this case are presented in Fig. 15. The SDFs calculated are quite different than that obtained for the case of only FP production (Fig. 13). The reason for this is very simple: the in-cascade cluster production mechanism provides continuous production of the smallest vacancy clusters at a rate which is independent of irradiation dose. For the case of only FP production the situation is qualitatively different since the mechanism providing void nucleation is reactions between two vacancies, whose concentration rapidly decreases as the cluster density increases with dose. Therefore, the void density reaches a near steady state value very rapidly giving rise to a regime of voids growth. However, under in-cascade nucleation conditions, the density of small voids continuously increases which restricts their capacity to grow. This situation is reproduced by the OKMC method. However, the OKMC SDF retains a tail at large sizes that is absent in the RT results. The origin of this difference is not clear at the time. 
Preliminary Comparison of Reaction Rate Theory and Object Kinetic

Monte Carlo Simulations

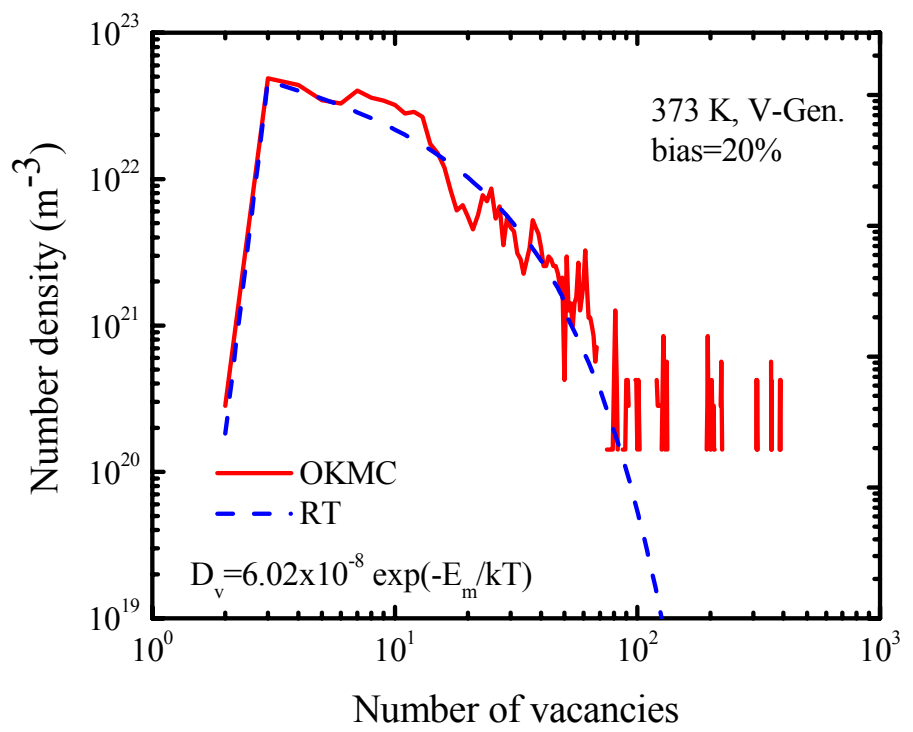

Fig. 15. Size distribution functions of voids at $0.01 \mathrm{dpa}$ calculated by the two methods for the case of in-cascade void generation, $E_{2 v}=0.2$ and $p=20 \%$.

The dose dependence of the void number density calculated using the RT and OKMC methods is presented in Fig. 16 for all three of the irradiation conditions that have been discussed.

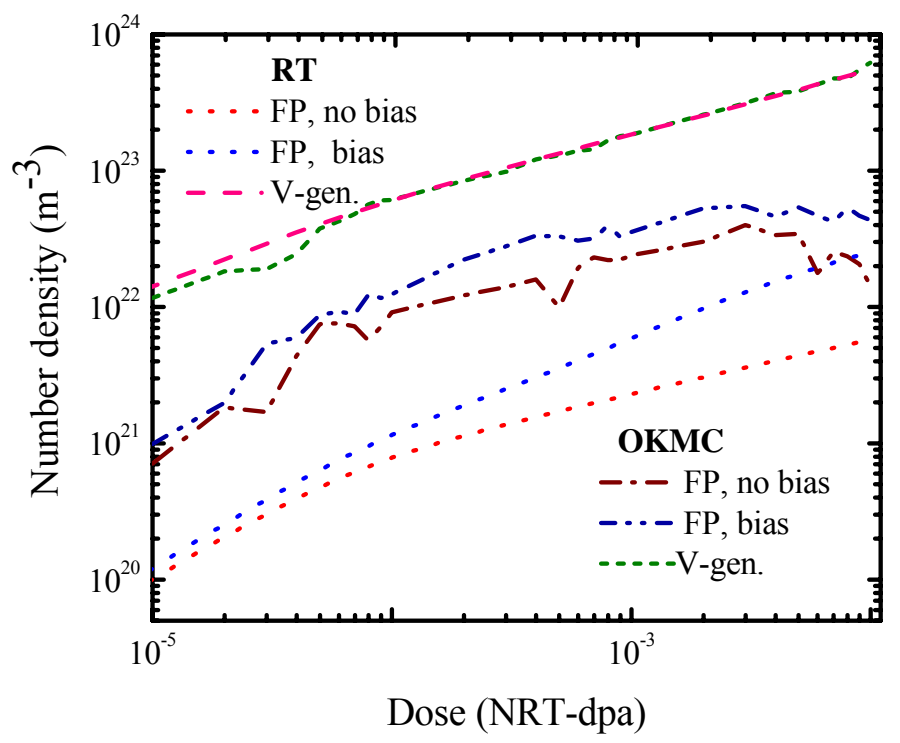

Fig. 16. Dose dependence of number density of voids calculated in all three cases using RT and OKMC methods. 
As can be seen from Fig. 16, there is a significant difference between the results obtained by the two methods for the two cases in which only FP production is considered. In particular, the void density calculated by OKMC is substantially smaller than that calculated from the RT for either zero or finite dislocation bias. Moreover, the void number density from the OKMC has a tendency to saturate at the higher doses while the RT value continues to increase with dose within the whole dose range calculated. However in the case of in-cascade void generation the agreement between the two methods is very good over the whole dose range. It shows that OKMC method is more reliable in the case when stable clusters directly produced under cascade irradiation conditions and, consequently, cluster density reaches a much higher value.

\subsubsection{OKMC calculations of swelling rate.}

Calculating the swelling rate for the case of FP production with fixed values of the void and dislocation sink strengths provides an additional consistency check on point defect accumulation between the two methods. The swelling rate given by Eq. (32) can be used as a basis of comparison of the swelling rate from the OKMC calculations in the void growth regime. For this comparison, 13 spherical absorbers with a radius of $0.4 \mathrm{~nm}$ were introduced in the simulation box to represent voids with a density of about $2 \times 10^{22} \mathrm{~m}^{-3}$. Another 13 spherical absorbers were introduced that had capture radii of $0.4 \mathrm{~nm}$ and $0.48 \mathrm{~nm}$ for vacancies and SIAs, respectively, to represent dislocations with an interstitial bias of $20 \%$. The simulation was designed to keep the void sink strength constant (i.e. the voids did not grow) during the irradiation while accounting for the total number of point defects captured by voids and dislocations. In this case, the sink strength of voids and dislocations are equal $\left(10^{14} \mathrm{~m}^{-2}\right)$ which should lead to a swelling rate of $1.3 \% / \mathrm{dpa}$ in accordance with Eq. (32). The calculations were run to a dose high enough to reach the steady state regime corresponding the specified swelling rate.

The calculated dose dependence of vacancy accumulation in voids and SIA accumulation in dislocations calculated is presented in Fig. 17. As can be seen from the plot, accumulation of SIAs in dislocations is positive already at very low doses, whereas accumulation of vacancies in the voids becomes positive at doses higher than about $2 \times 10^{-4} \mathrm{dpa}$. This difference is related to the difference between the vacancy and SIA diffusivity. However, at doses higher than $2 \times 10^{-4}$ dpa, the vacancy concentration also reaches a steady state level and the accumulation of point defects 
in voids and dislocations follow one other. Note that vacancy accumulation in voids is smaller than that of SIA accumulation in dislocations over the whole dose range. This difference is accounted for by the higher steady state vacancy concentration accumulated in the simulation box.

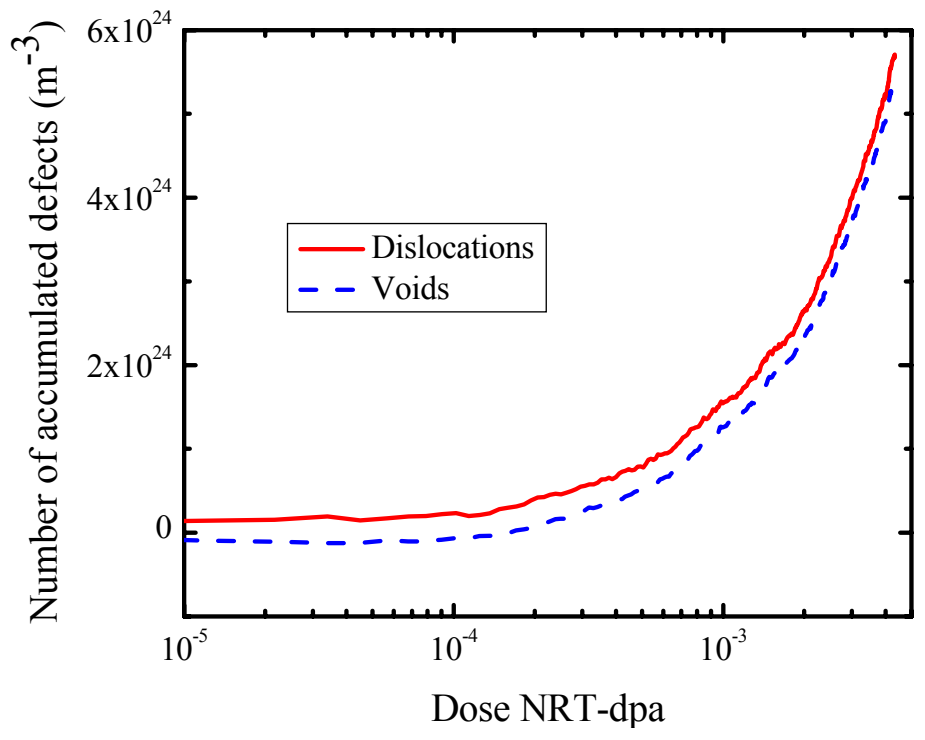

Fig. 17. Dose dependence of point defect accumulation in voids of a given density and size and in dislocations of a given density.

Differentiating the accumulation curves presented in Fig. 17 one can calculate the effective instantaneous swelling rate, i.e. the net rate of accumulation of vacancies and SIAs in voids and dislocations, respectively. The results of this differentiation are presented in Fig. 18. After the vacancy concentration reaches steady state, the average swelling rate from the OKMC is equal to about 1.5\%/dpa which is somewhat higher than the value predicted above from Eq. (32). However the fluctuations in the instantaneous value of the swelling rate are several times larger than the average value. There are several possible effects that may be responsible for such a variation in the swelling rate, e.g. differences in the vacancy and SIA diffusivity lead to a separation in time for the capture of newly-introduced SIAs and vacancies by the sinks. The box size limitations on the sink density also may be responsible for the strong variation in the swelling rate. 

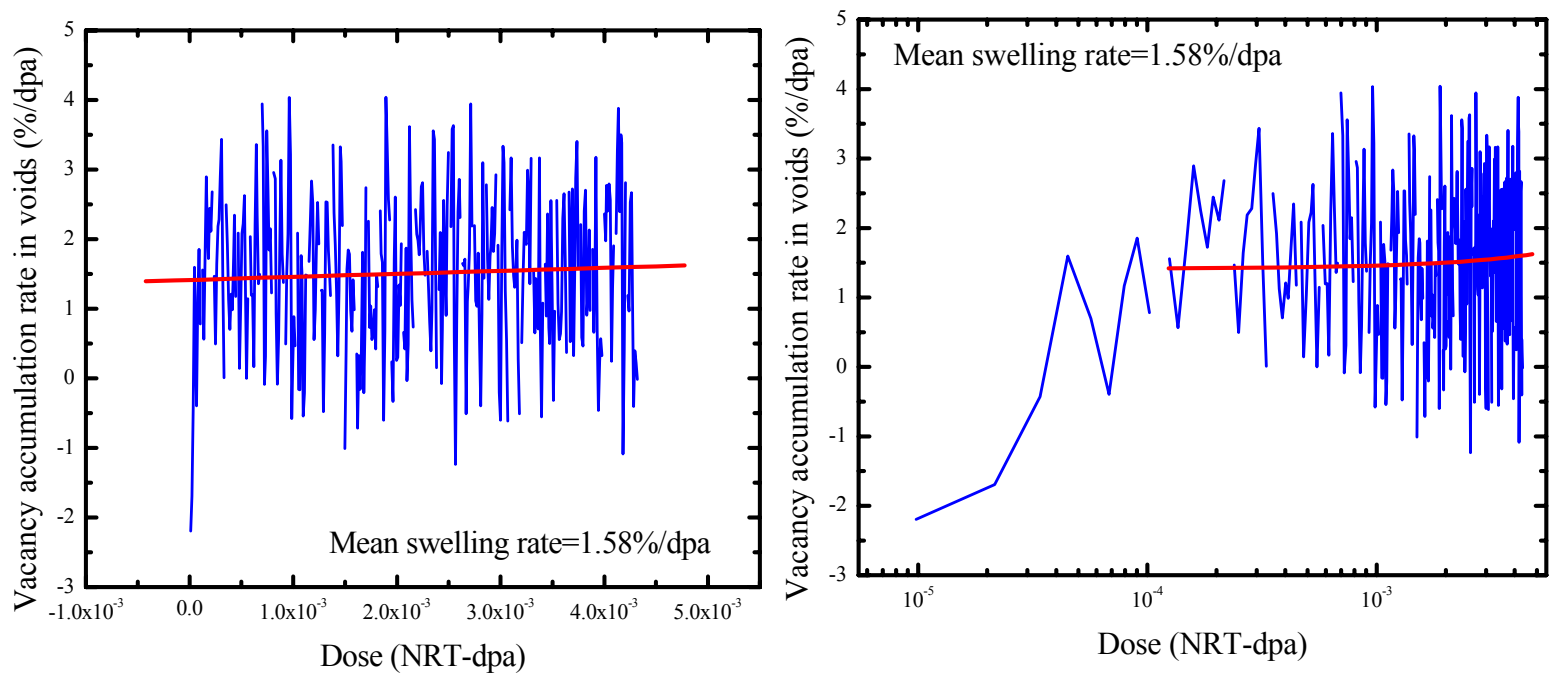

Fig. 18. Dose dependence of the swelling rate based on the instantaneous rate of vacancy accumulation in voids.

\section{SUMMARY AND FURTHER WORK}

The comparison between the rate theory and object kinetic Monte Carlo methods has found a persistent difference between the results obtained for the two codes, particularly for the case of only Frenkel pair production. The primary basis of this difference appears to be that, for a given simulation condition, the OKMC obtains a slightly higher vacancy concentration. This leads to somewhat greater vacancy accumulation in voids, as indicated in the void SDFs and in the swelling rate comparison. There are several possible reasons for the observed differences that have been discussed above. Some follow directly from the differences between the two methods listed in Table 1: statistical variations in the OKMC, effects related to OKMC box size (for example the steady state interstitial concentration is much less than one in the simulation cell), and subtle differences in the way the point defects are introduced by the two methods' source term. In the case of FP production, only homogeneous void nucleation occurs, so the nucleation rate is very sensitive to the effective vacancy supersaturation. The accuracy of the vacancy supersaturation may suffer when the absolute point defect densities (number per box) are relatively low in the OKMC simulation. The agreement between the two methods is much better for irradiation conditions with in-cascade vacancy cluster formation. In this case, the cascadedriven nucleation of voids gives a higher density, which improves the statistics in the OKMC simulation. Finally, specific simulations must be structured very carefully to ensure that exactly 
the same problem is being solved by the two methods. In the end, it is not as simple as first thought to cross-compare these two methods.

Further work is underway to resolve the remaining differences using several approaches. One part involves setting up a series of simulations to isolate individual processes, e.g. matrix recombination point defects vs. their absorption at sinks, to verify unit mechanisms. Further investigation of statistical variations as illustrated by Figs. 9 and 10 planned. A second statistical source of differences being investigated is the impact of accessible sink densities based on the OKMC box size. Once the final cross validation is completed on the sample problems, the two methods will be applied to a broader selection of problems. These will include the addition of incascade interstitial clustering, point defect cluster mobility, and variations in the irradiation temperature and displacement rate. This will help define irradiation regimes in which one of the two methods may be preferred over the other. For example, current box size limitations imply that it may be difficult to use the OKMC at low displacement rates and high temperatures. However, the OKMC does a better job of accounting for spatial correlations in the primary damage source term under cascade damage conditions.

\section{REFERENCES}

1. M. Kiritani, J. Phys. Soc. Japan 35 (1973) 95.

2. N. M. Ghoniem and S. Sharafat, J. Nucl. Mater. 92 (1980) 121.

3. S. I. Golubov, A. M. Ovcharenko, A. V. Barashev, and B. N. Singh, Philos. Mag. A 81 (2001) 643.

4. M. P. Surh, J. B. Sturgeon, W.G. Wolfer, J. Nucl. Mater. 325 (2004) 44.

5. N. Metropolis, A.W. Rosenbluth, M.N. Rosenbluth, A.H. Teller and E. Teller, J. Chem. Phys. 21 (1953) 1087.

6. W. A. Coghlan and L. K. Mansur, J. Nucl. Mater. 108\&109 (1982) 246.

7. J. L. Katz and H. Wiedersich, J. Chem. Phys. 55 (1973) 1414.

8. S. I. Golubov, S. I., Phys. Metals Metallogr. (USSR) 52 (1981) 86.

9. C. Domain, C. S. Becquart, and L. Malerba, J. Nucl. Mater. 335 (2004) 121.

10. W. M. Young and E. W. Elcock, Proc. Phys. Soc. 89 (1966) 735.

11. B. Bortz, M. H. Kalos, J. L. Lebowitz, J. Comp. Phys. 17 (1975) 10.

12. L. K. Mansur, "Mechanisms and Kinetics of Radiation Effects in Metals and Alloys," Kinetics of Nonhomogeneous Processes, Wiley-Interscience, New York, 1987, pp. 377-463. 
Preliminary Comparison of Reaction Rate Theory and Object Kinetic Monte Carlo Simulations

13. H. L. Heinisch, B. N. Singh, and S. I. Golubov, J. Computer-Aided Mat. Design, 6 (1999) 277.

14. S. I. Golubov, B. N. Singh, and H. Trinkaus, Phil. Mag. A 81 (2001) 2533. 


\section{Distribution}

1. T. D. Burchell

2. W. R. Corwin

3. S. R. Greene

4. D. T. Ingersoll

5. Y. Katoh

6. L. K. Mansur

7. J. R. Morris

8. R. K. Nanstad

9. D. M. Nicholson

10. Yu. N. Osetskiy
11. B. Radhakrishnan

12. R. A. Raschke

13. L. L. Snead

14. R. E. Stoller

15. S. J. Zinkle

16. Cathy Barnard, Idaho National Engineering and Environmental Laboratory, P.O. Box 1625, Idaho Falls, Idaho 83415-3750

17. C. S. Becquart, LMPGM, UMR-CNRS 8517, Université de Lille I, 59655 Villeneuve D'Ascq, France

18. Trevor Cook, NE-20/Germantown Building, Office of Advanced Nuclear Research, U.S. Department of Energy, 1000 Independence Avenue, S.W., Washington, DC 20585-1290

19. S. Domain, EDF-R\&D, Dept. MMC, Les Renardières, Moret-sur-Loing, France

20. Susan Lesica, NE-20/Germantown Building, Office of Advanced Nuclear Research, U.S. Department of Energy, 1000 Independence Avenue, S.W., Washington, DC 20585-1290

21. Thomas J. O'Conner, NE-20/Germantown Building, Office of Advanced Nuclear Research, U.S. Department of Energy, 1000 Independence Avenue, S.W., Washington, DC 20585-1290

22. Rafael Soto, Idaho National Engineering and Environmental Laboratory, P.O. Box 1625, Idaho Falls, Idaho 83415-3750

23. Robert Versluis, NE-20/Germantown Building, Office of Advanced Nuclear Research, U.S. Department of Energy, 1000 Independence Avenue, S.W., Washington, DC 205851290 